Supporting Information

\title{
Total Synthesis of Nominal ent-Chlorabietol B
}

Yulong Li, Zhezhe Xu, Zhipeng Xie, Xingchao Guan, and Zhixiang Xie*

State Key Laboratory of Applied Organic Chemistry, College of Chemistry and Chemical

Engineering, Lanzhou University, Lanzhou, 730000, China

\section{Contents}

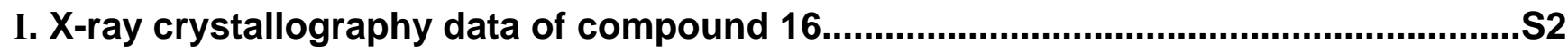

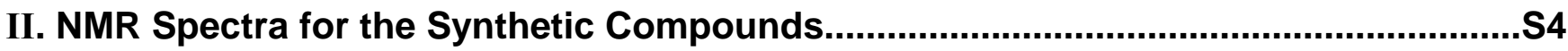

III. NMR data comparison of synthetic sample and natural product............................ 223 


\section{X-ray crystallography data of compound 16}

Preparation of crystal: Compound $\mathbf{1 6}$ was dissolved in a solution of petroleum ether and ethyl acetate $(\mathrm{PE}: \mathrm{EA}=8: 1)$ to a subsaturating level. Then, transfer the solution to a clean container with a large surface and cover it not too tightly (aluminum foil with some punched holes seems to work very well). The solution evaporates for a week with any disturbance, and the crystal of compound $\mathbf{1 6}$ was achieved. The X-ray single-crystal determination of $\mathbf{1 6}$ was performed on an Agilent SuperNova single crystal X-ray diffractometer.

\section{X-ray crystallography of compound 16}
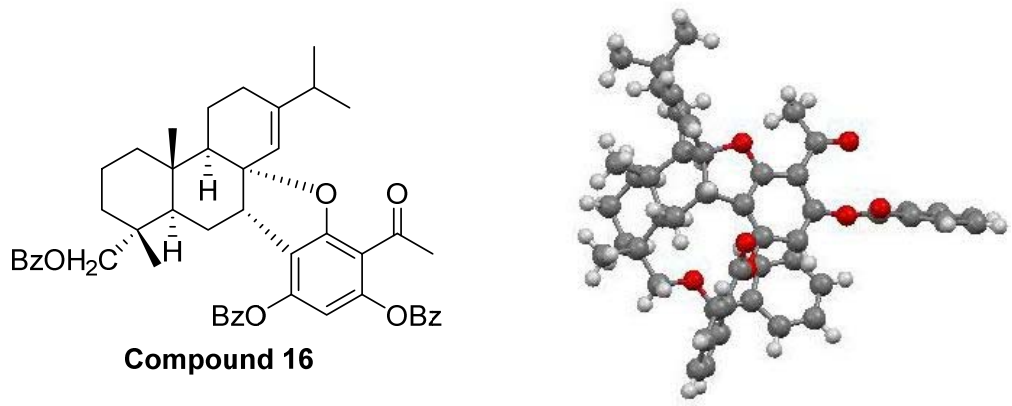

X-ray crystallography of 16

Table S1 Crystal data and structure refinement for liyl 1118.

\begin{tabular}{|c|c|}
\hline Identification code & liyl_1118 \\
\hline Empirical formula & $\mathrm{C}_{49} \mathrm{H}_{50} \mathrm{O}_{8}$ \\
\hline Formula weight & 766.89 \\
\hline Temperature/K & $293.10(10)$ \\
\hline Crystal system & monoclinic \\
\hline Space group & $\mathrm{P} 2_{1}$ \\
\hline $\mathrm{a} / \AA$ & $10.5804(5)$ \\
\hline $\mathrm{b} / \AA$ & $17.8970(6)$ \\
\hline $\mathrm{c} / \AA ̊$ & $12.2550(5)$ \\
\hline$\alpha /^{\circ}$ & 90 \\
\hline$\beta /{ }^{\circ}$ & $115.276(5)$ \\
\hline$\gamma /{ }^{\circ}$ & 90 \\
\hline Volume $/ \AA^{3}$ & 2098.39(17) \\
\hline $\mathrm{Z}$ & 2 \\
\hline$\rho_{\text {calc }} \mathrm{g} / \mathrm{cm}^{3}$ & 1.214 \\
\hline$\mu / \mathrm{mm}^{-1}$ & 0.655 \\
\hline $\mathrm{F}(000)$ & 816.0 \\
\hline Crystal size $/ \mathrm{mm}^{3}$ & $0.11 \times 0.07 \times 0.02$ \\
\hline Radiation & $\operatorname{CuK} \alpha(\lambda=1.54184)$ \\
\hline $2 \Theta$ range for data collection ${ }^{\circ}$ & 7.978 to 133.198 \\
\hline Index ranges & $-12 \leq \mathrm{h} \leq 11,-21 \leq \mathrm{k} \leq 21,-13 \leq 1 \leq 14$ \\
\hline Reflections collected & 13000 \\
\hline Independent reflections & $6954\left[\mathrm{R}_{\mathrm{int}}=0.0241, \mathrm{R}_{\text {sigma }}=0.0325\right]$ \\
\hline Data/restraints/parameters & $6954 / 1 / 519$ \\
\hline Goodness-of-fit on $\mathrm{F}^{2}$ & 1.061 \\
\hline Final $R$ indexes $[\mathrm{I}>=2 \sigma(\mathrm{I})]$ & $\mathrm{R}_{1}=0.0464, \mathrm{wR}_{2}=0.1148$ \\
\hline Final $\mathrm{R}$ indexes [all data] & $\mathrm{R}_{1}=0.0512, \mathrm{wR}_{2}=0.1205$ \\
\hline Largest diff. peak/hole / e $\AA^{-3}$ & $0.17 /-0.21$ \\
\hline Flack parameter & $-0.07(9)$ \\
\hline
\end{tabular}




\section{ORTEP of the compound 16:}

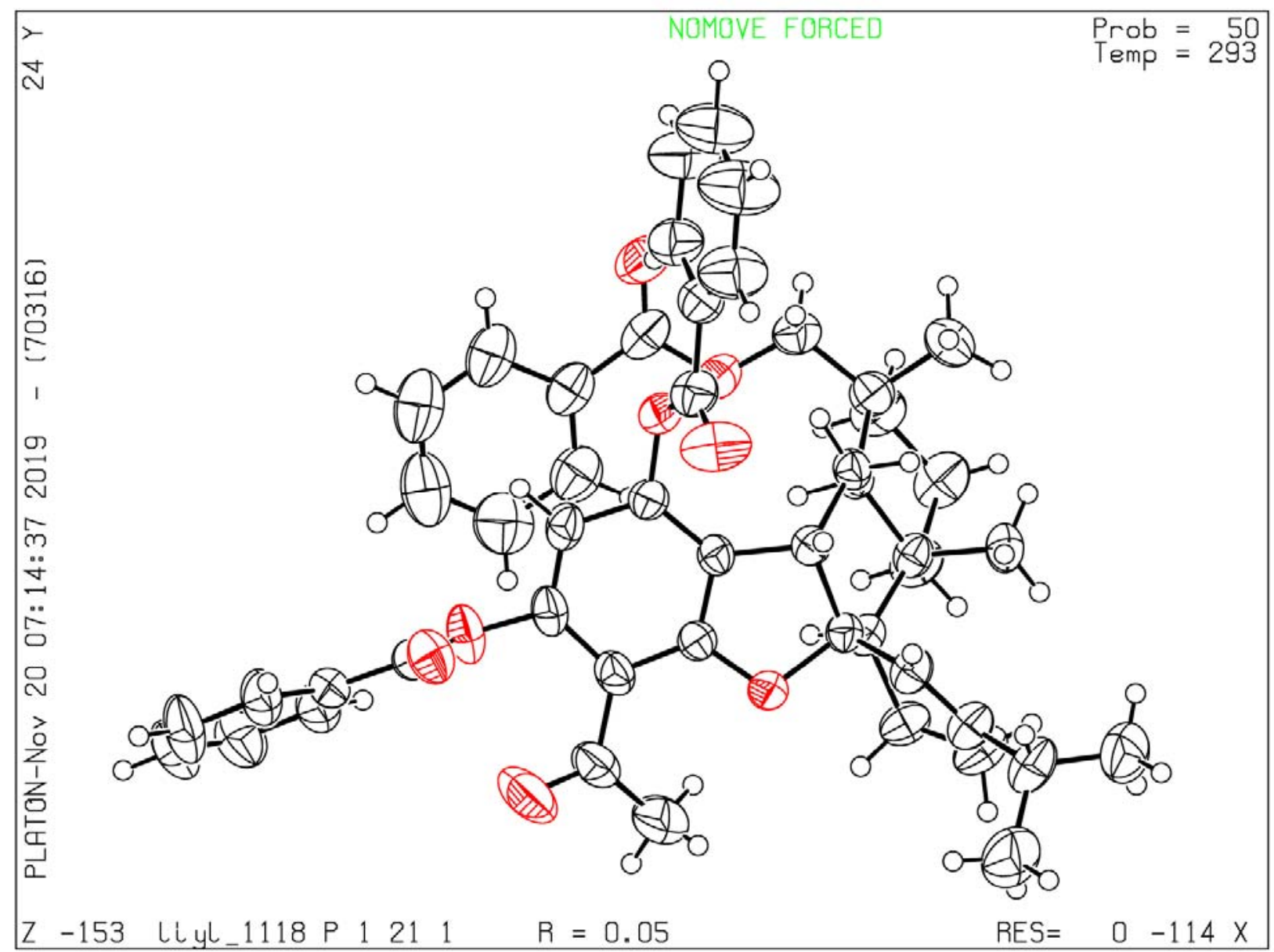

Cystallographic data is available free of charge from the Cambridge Crystallographic Data Centre (https://summary.ccdc.cam.ac.uk/structure-summary-form under deposition number 1972686). 


\section{NMR Spectra for the Synthetic Compounds}
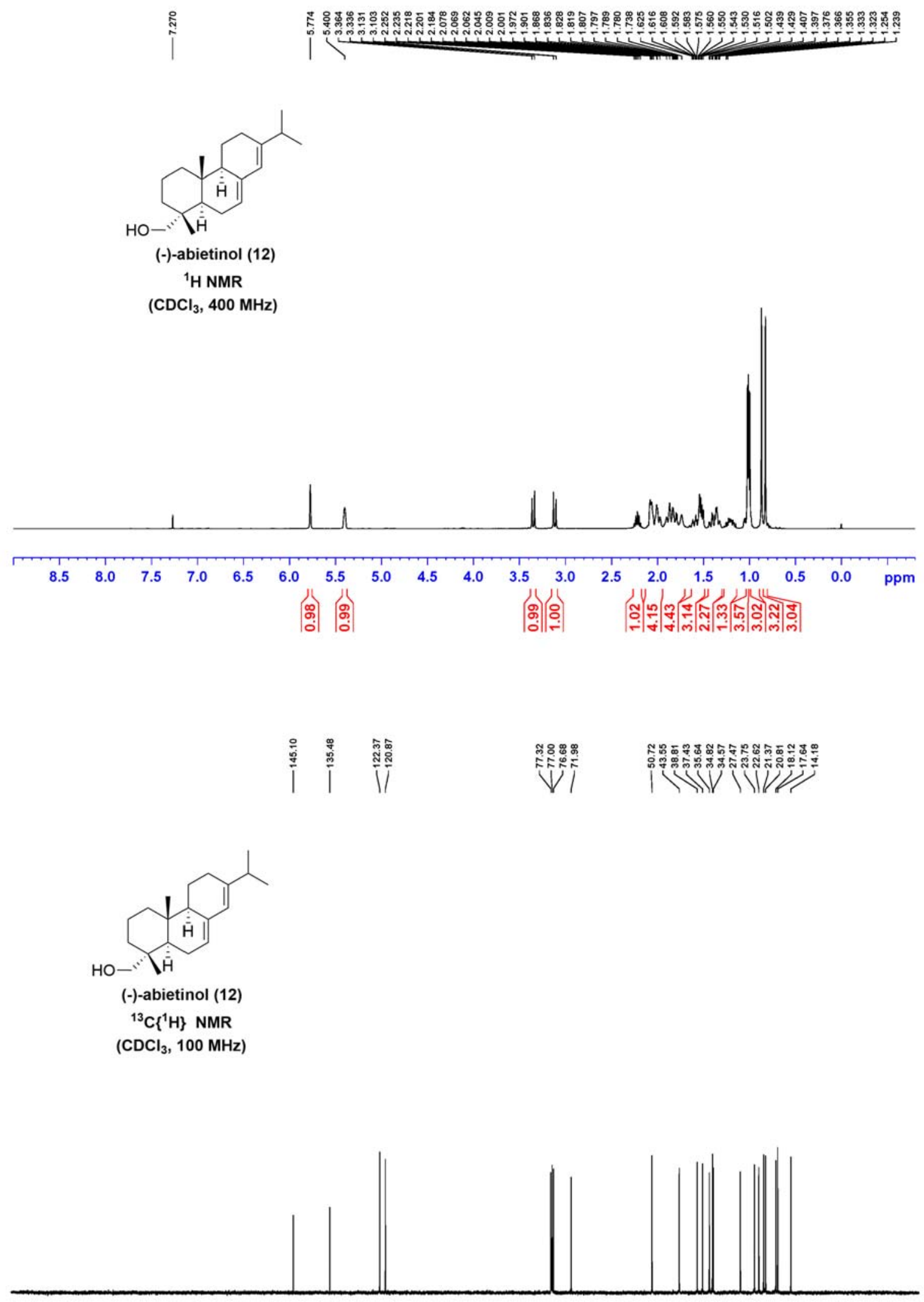

$\begin{array}{lllllllllllllllllllllll}210 & 200 & 190 & 180 & 170 & 160 & 150 & 140 & 130 & 120 & 110 & 100 & 90 & 80 & 70 & 60 & 50 & 40 & 30 & 20 & 10 & 0 & \mathrm{ppm}\end{array}$ 

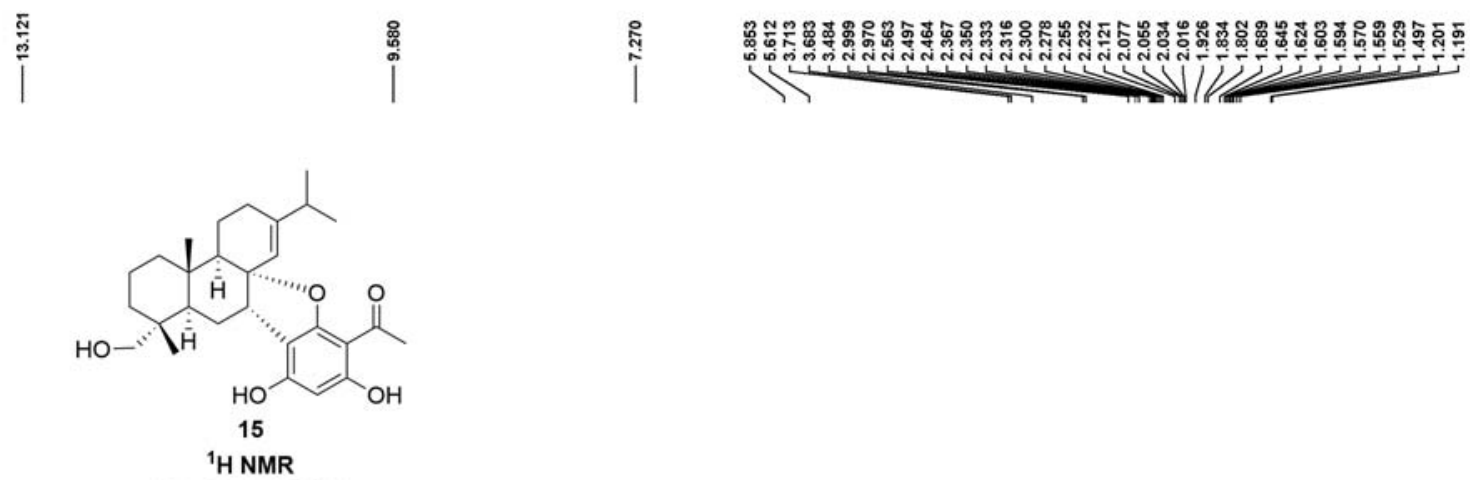

$\left(\mathrm{CDCl}_{3}, 400 \mathrm{MHz}\right)$

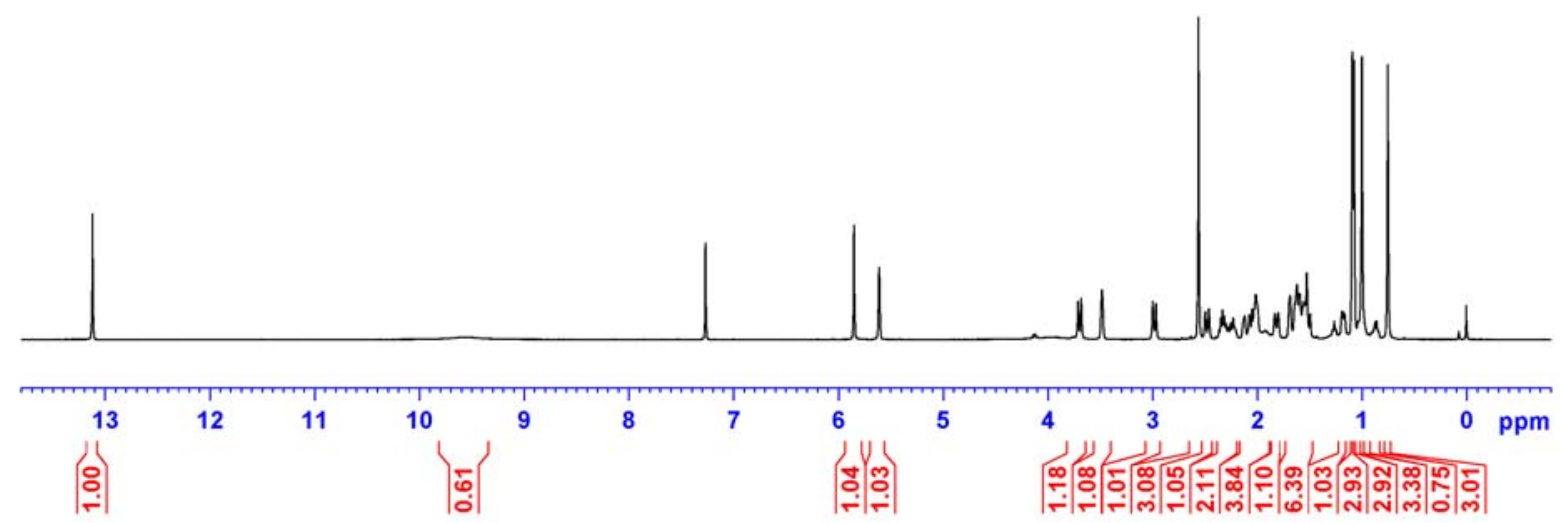

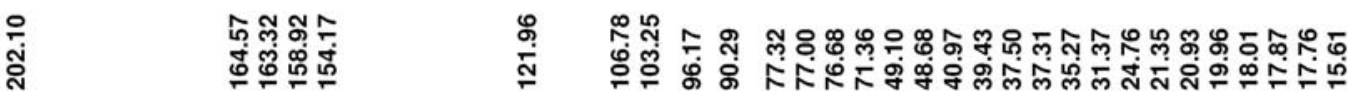

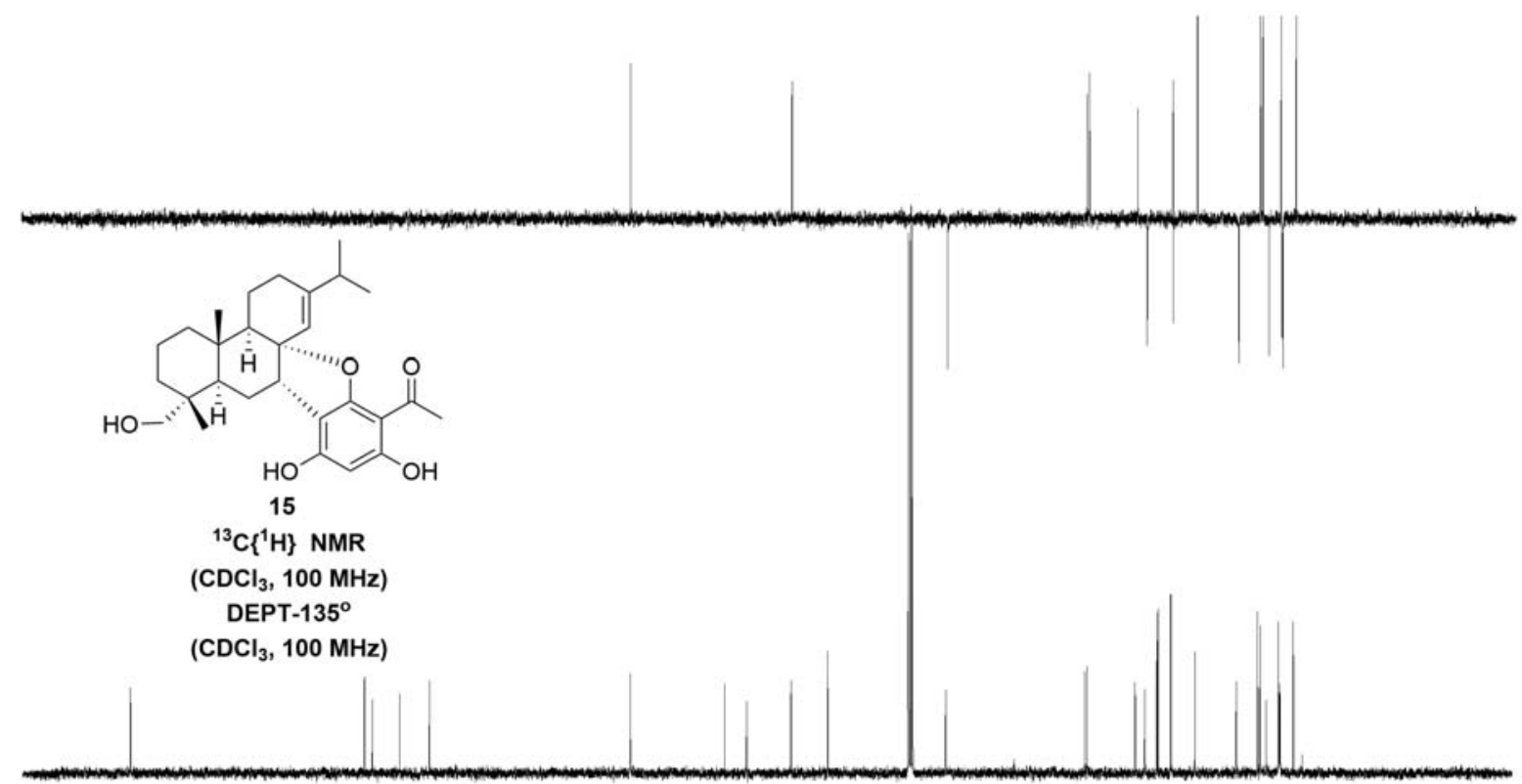

$\begin{array}{lllllllllllllllllllllll}210 & 200 & 190 & 180 & 170 & 160 & 150 & 140 & 130 & 120 & 110 & 100 & 90 & 80 & 70 & 60 & 50 & 40 & 30 & 20 & 10 & 0 & \mathrm{ppm}\end{array}$ 

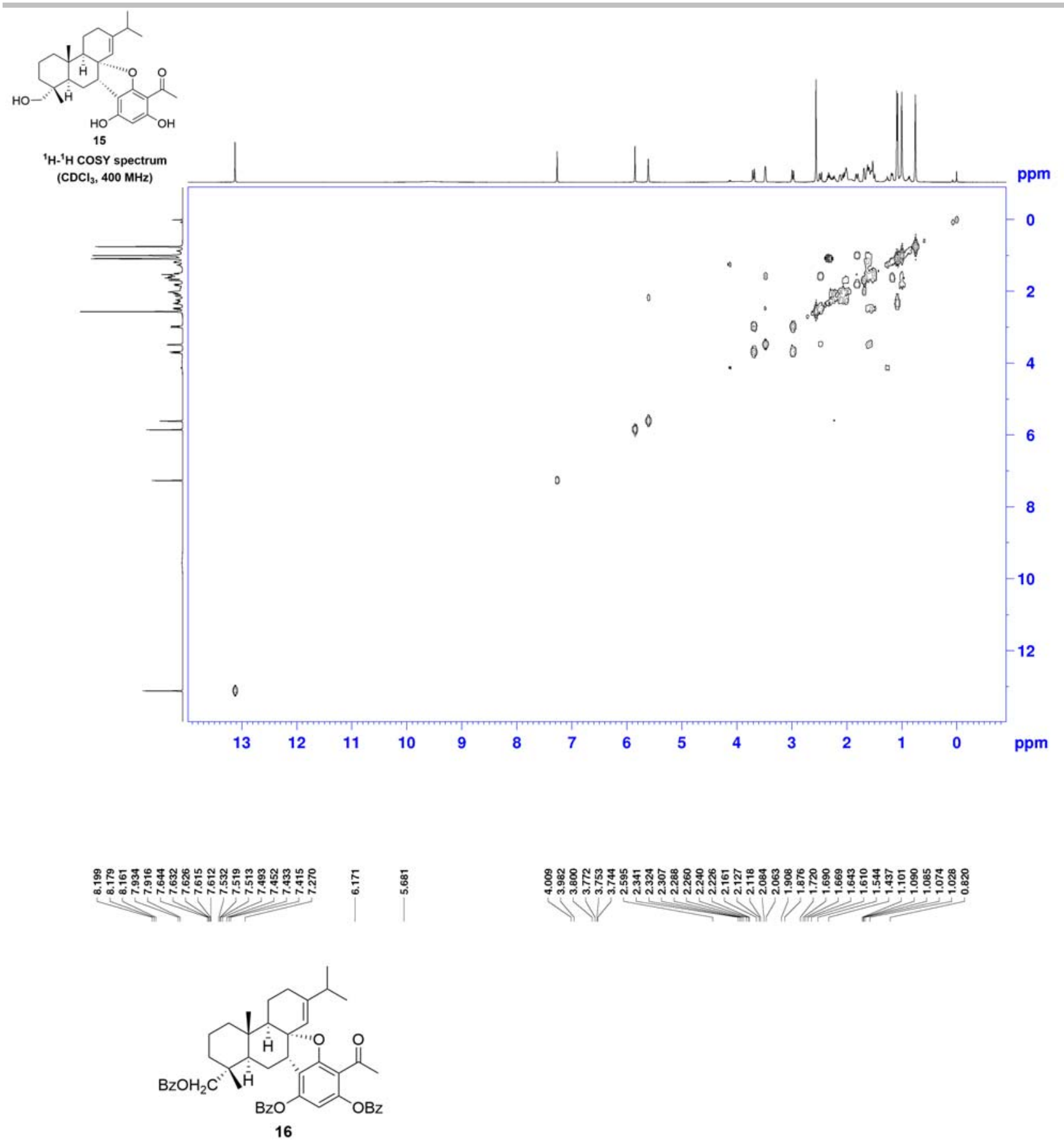

${ }^{1} \mathrm{H}$ NMR

$\left(\mathrm{CDCl}_{3}, 400 \mathrm{MHz}\right)$

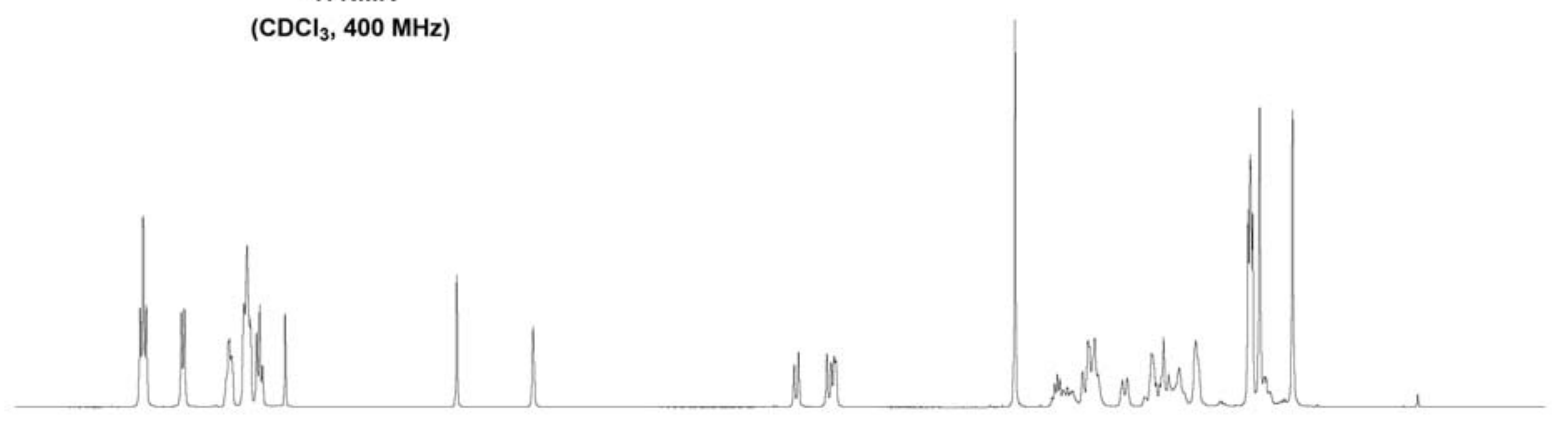

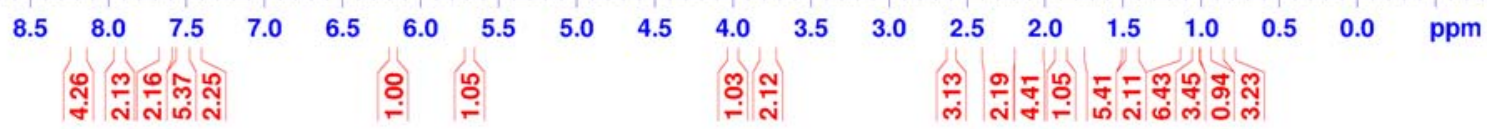




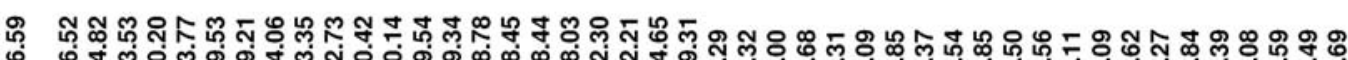

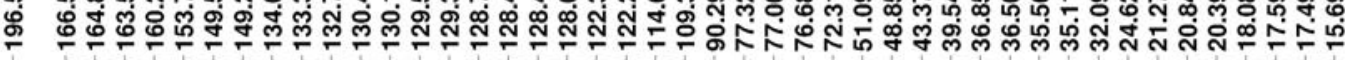

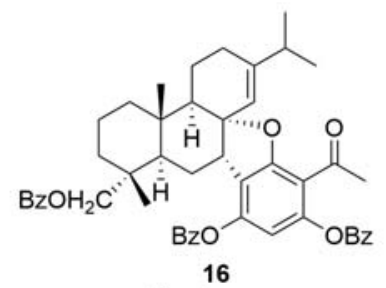

${ }^{13} \mathrm{C}\left\{{ }^{1} \mathrm{H}\right\}$ NMR $\left(\mathrm{CDCl}_{3}, 100 \mathrm{MHz}\right)$

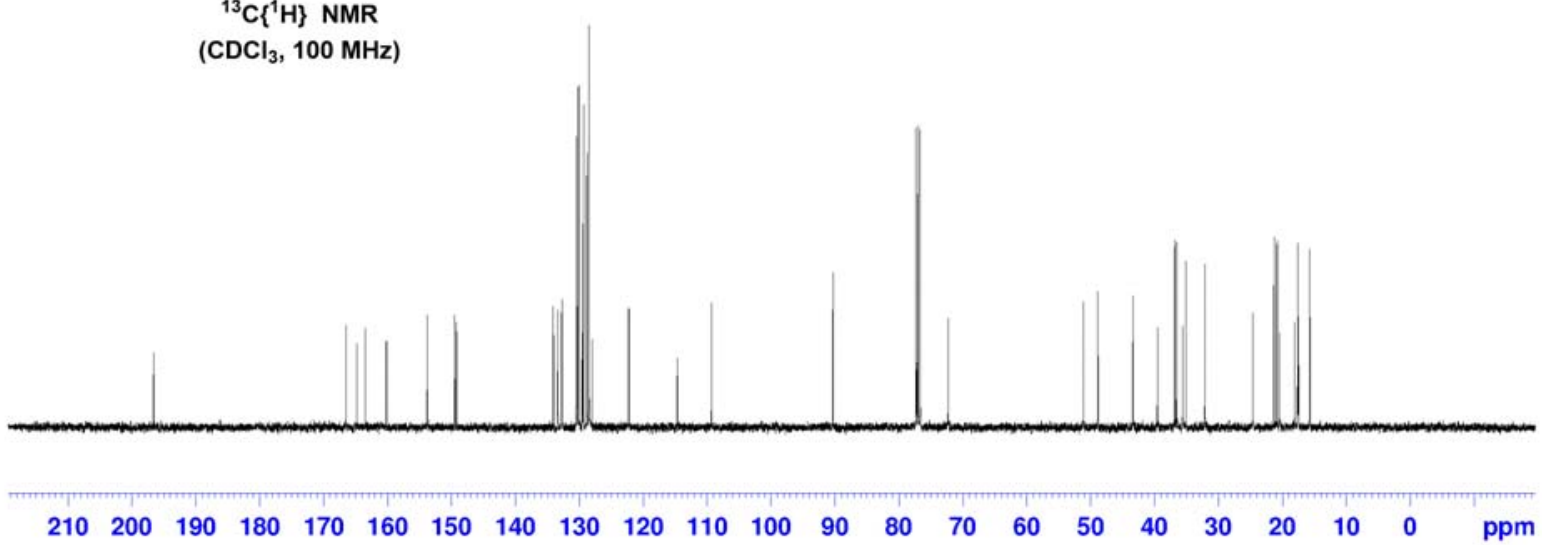

\section{太}

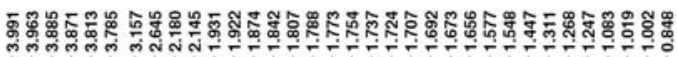

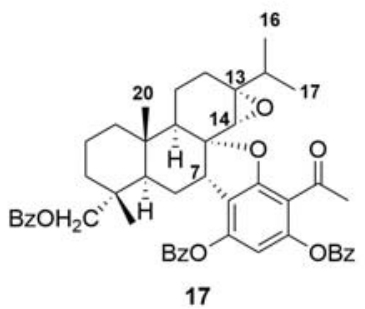

${ }^{1} \mathrm{H}$ NMR

$\left(\mathrm{CDCl}_{3}, 400 \mathrm{MHz}\right)$

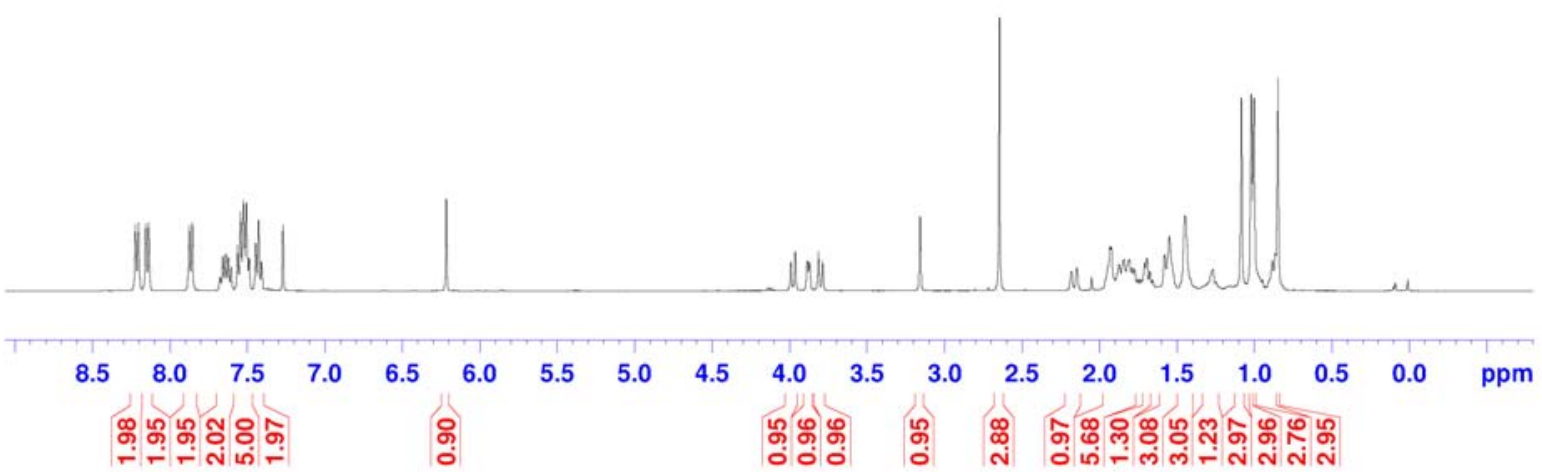




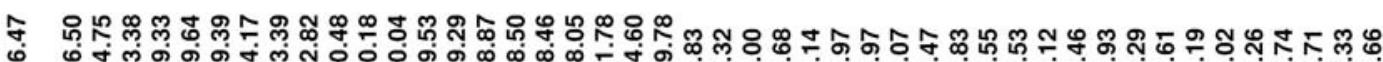

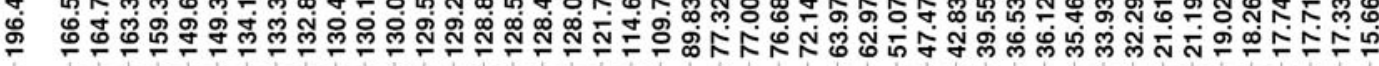

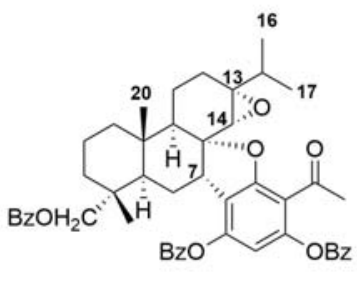

17

${ }^{13} \mathrm{C}\left\{{ }^{1} \mathrm{H}\right\}$ NMR

$\left(\mathrm{CDCl}_{3}, 100 \mathrm{MHz}\right)$

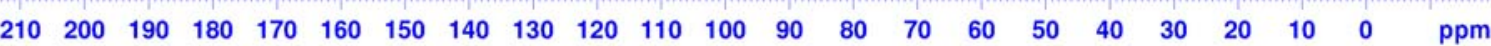

|y|20190424-1HNOF:

STANDARD PROTON PARAMETERS

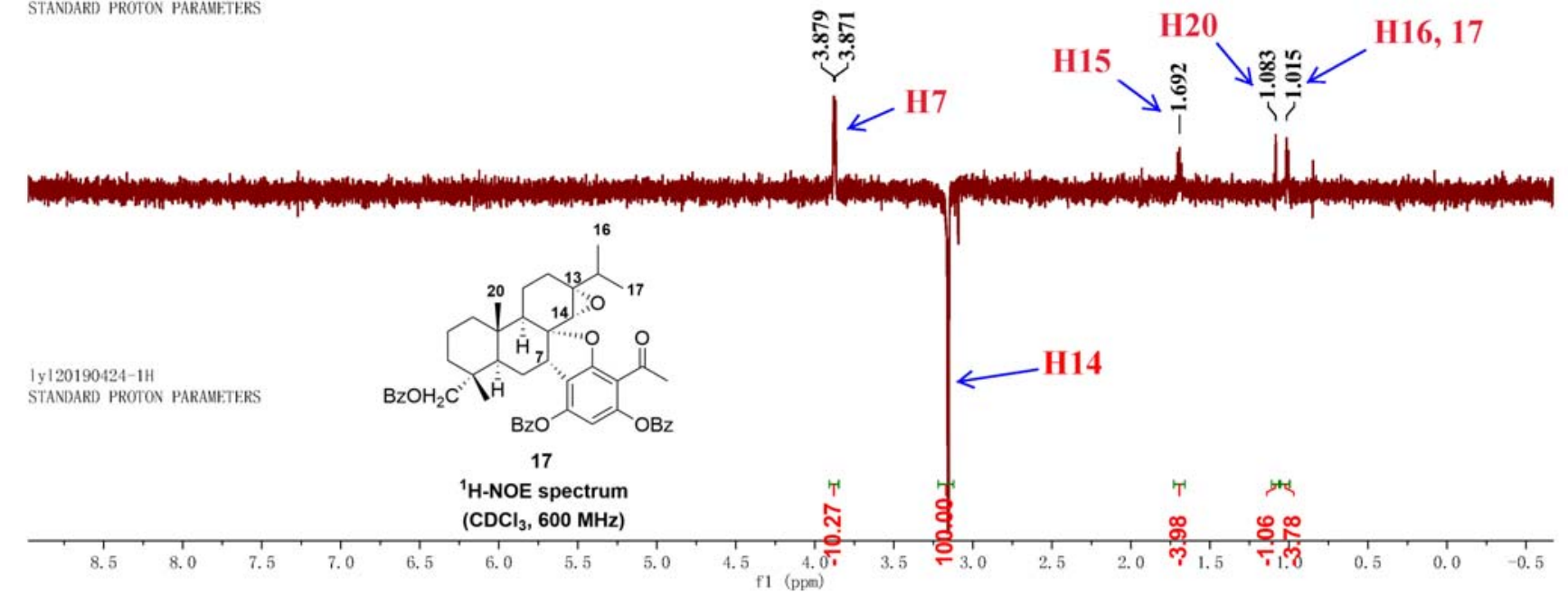

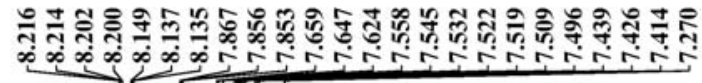

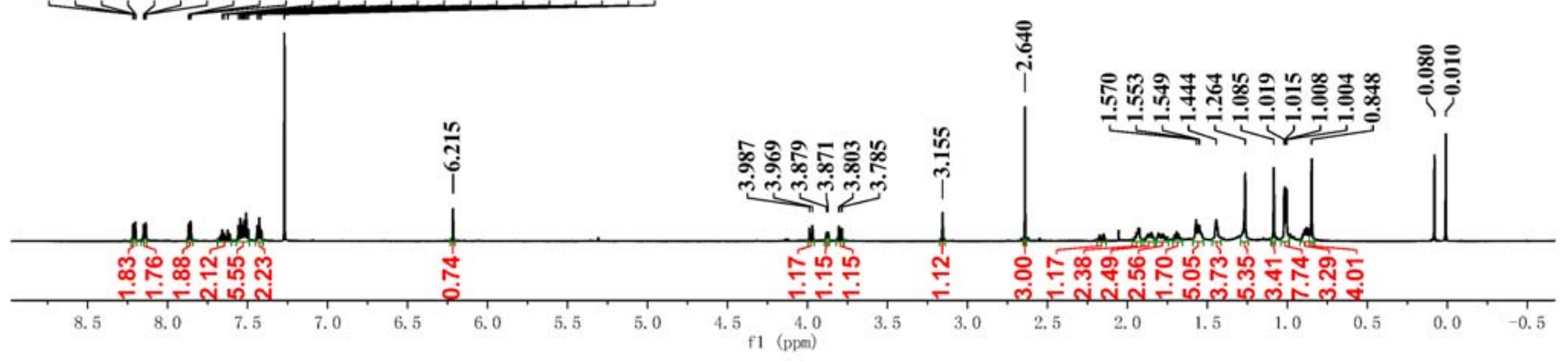



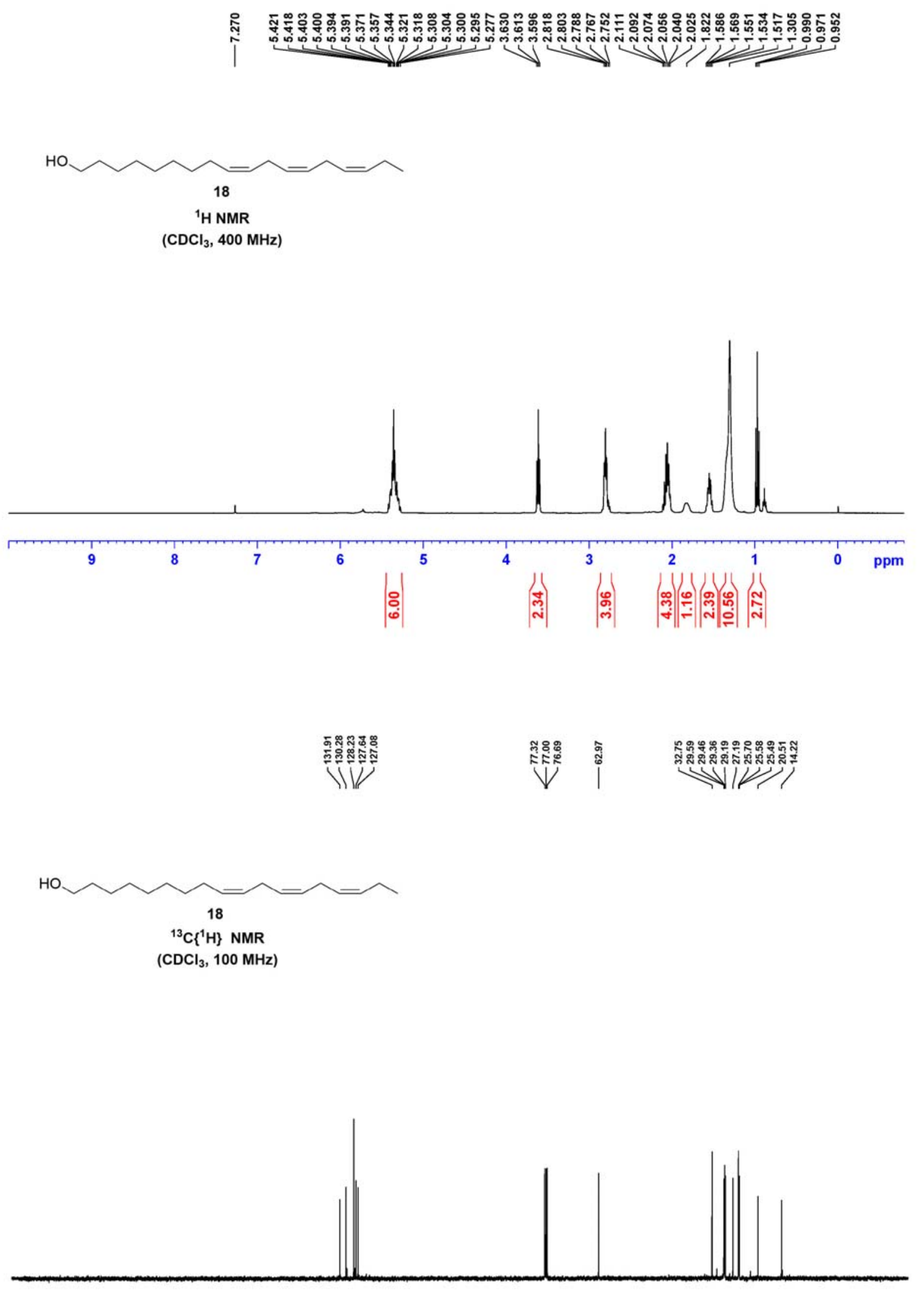

$\begin{array}{lllllllllllllllllllllll}210 & 200 & 190 & 180 & 170 & 160 & 150 & 140 & 130 & 120 & 110 & 100 & 90 & 80 & 70 & 60 & 50 & 40 & 30 & 20 & 10 & 0 & \mathrm{ppm}\end{array}$ 

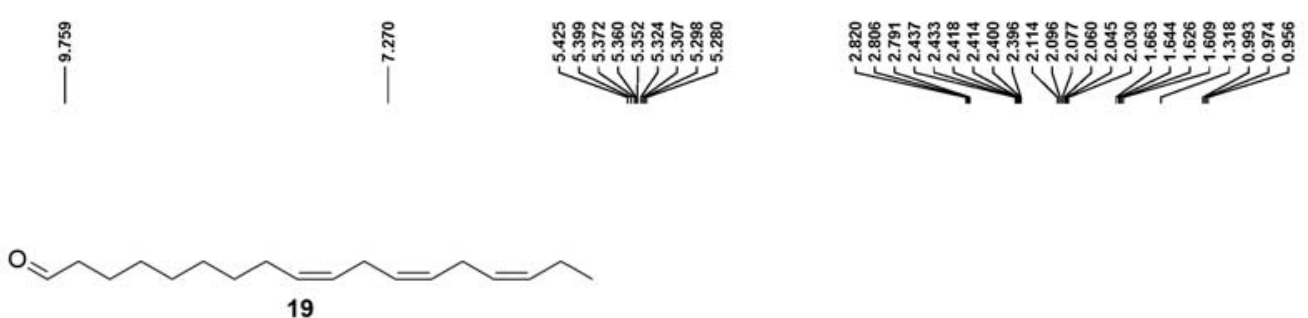

${ }^{1} \mathrm{H}$ NMR

$\left(\mathrm{CDCl}_{3}, 400 \mathrm{MHz}\right)$
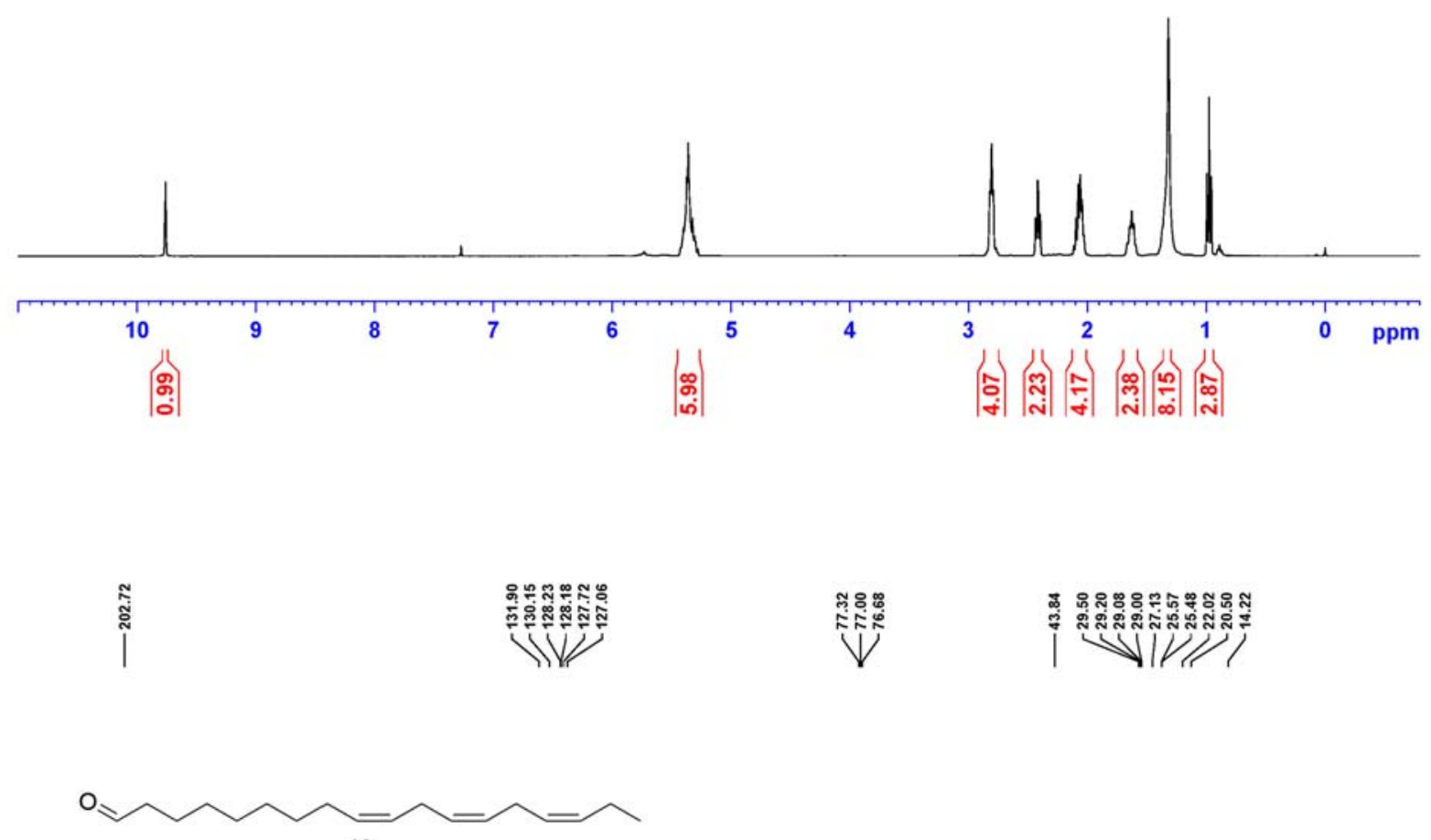

19

${ }^{13} \mathrm{C}\left\{{ }^{1} \mathrm{H}\right\}$ NMR

$\left(\mathrm{CDCl}_{3}, 100 \mathrm{MHz}\right)$

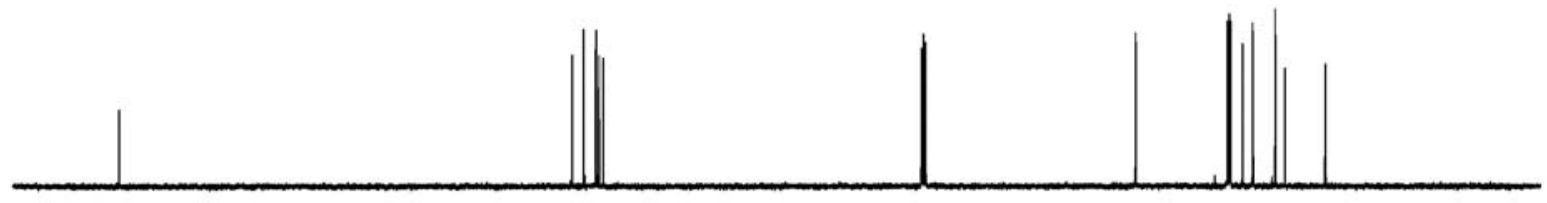

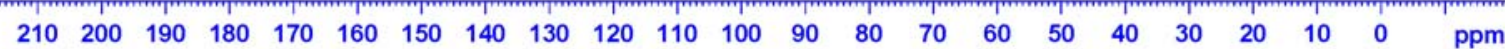



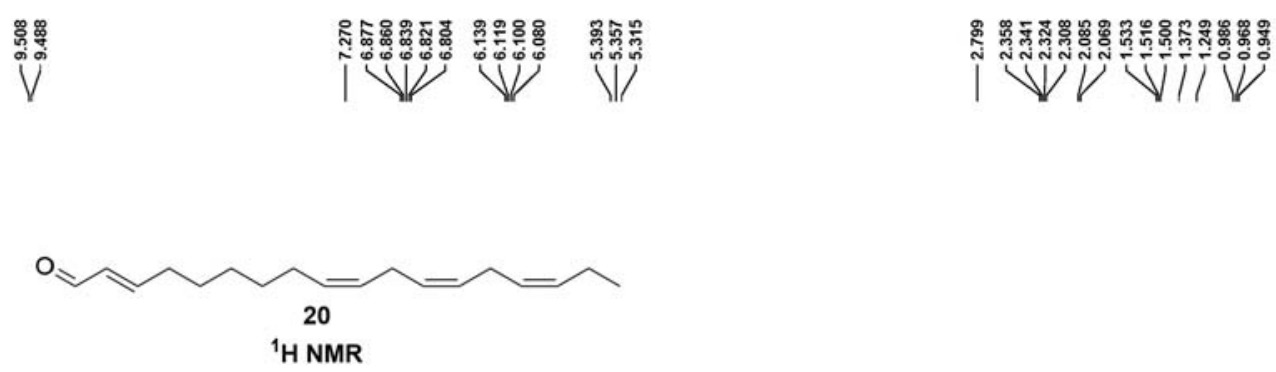

$\left(\mathrm{CDCl}_{3}, 400 \mathrm{MHz}\right)$
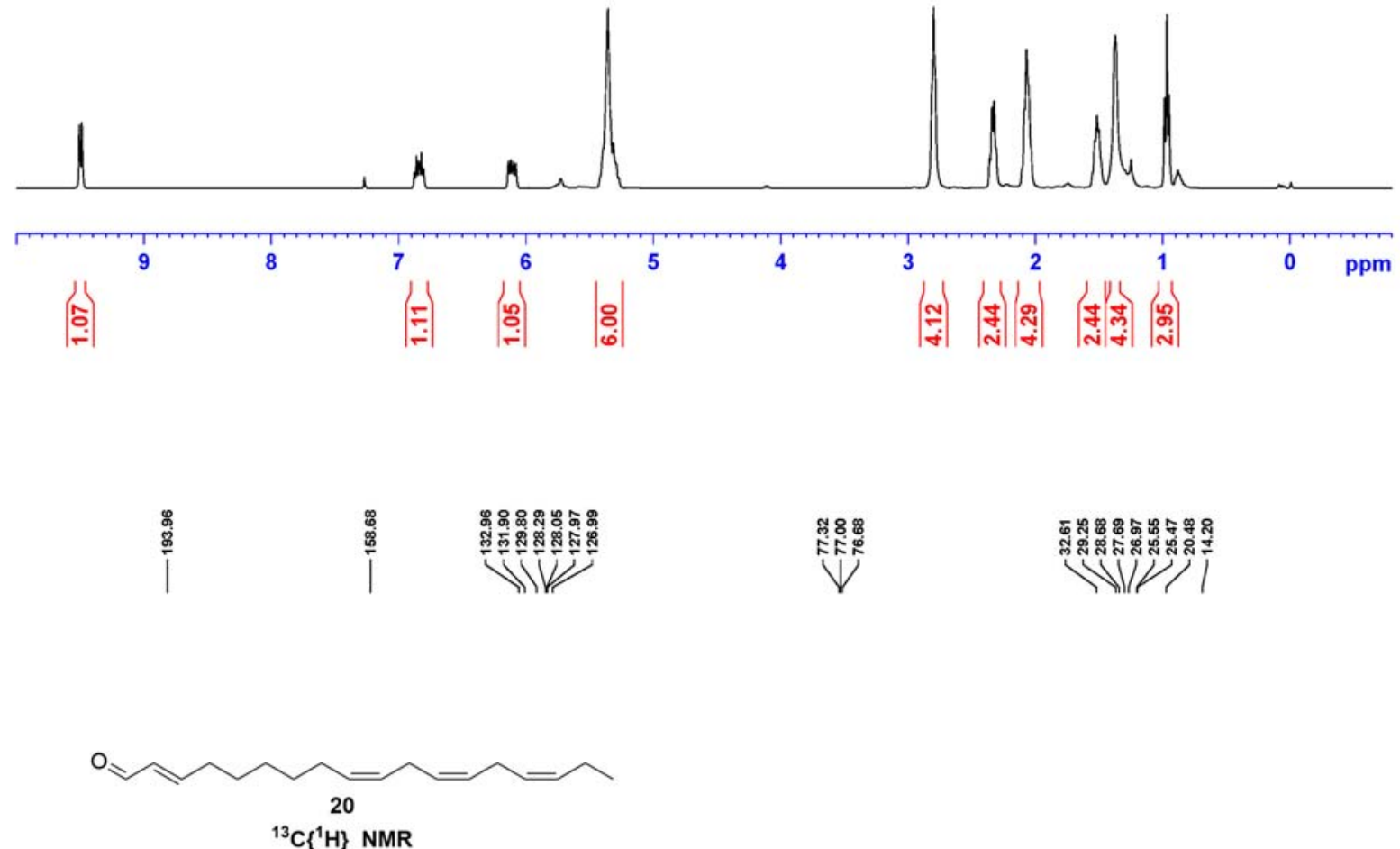

$\left(\mathrm{CDCl}_{3}, 100 \mathrm{MHz}\right)$

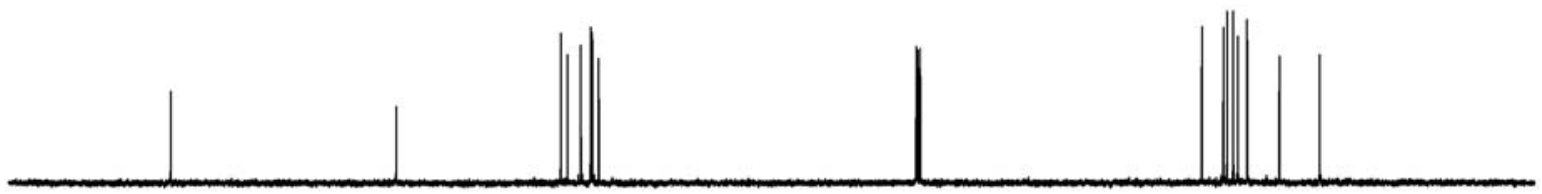

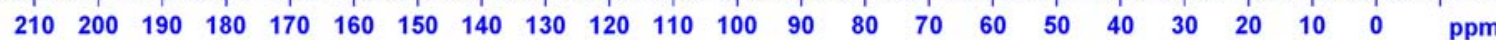



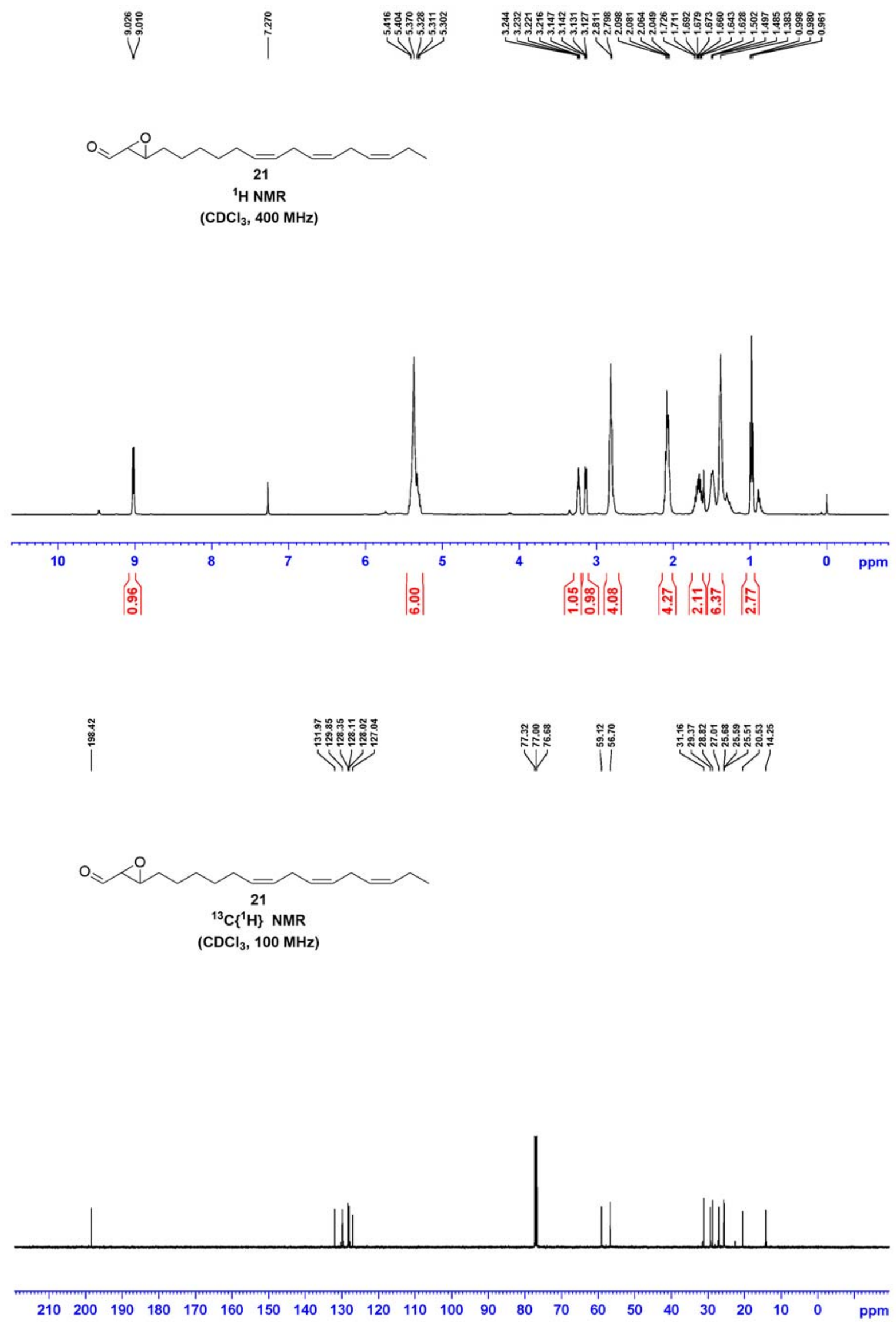


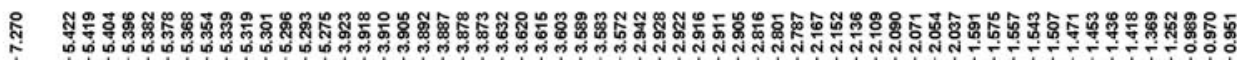

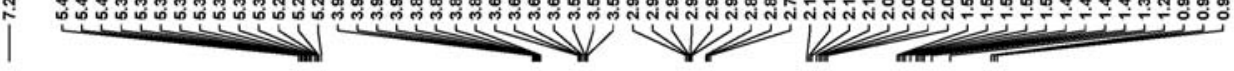

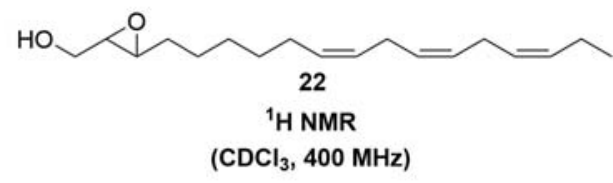
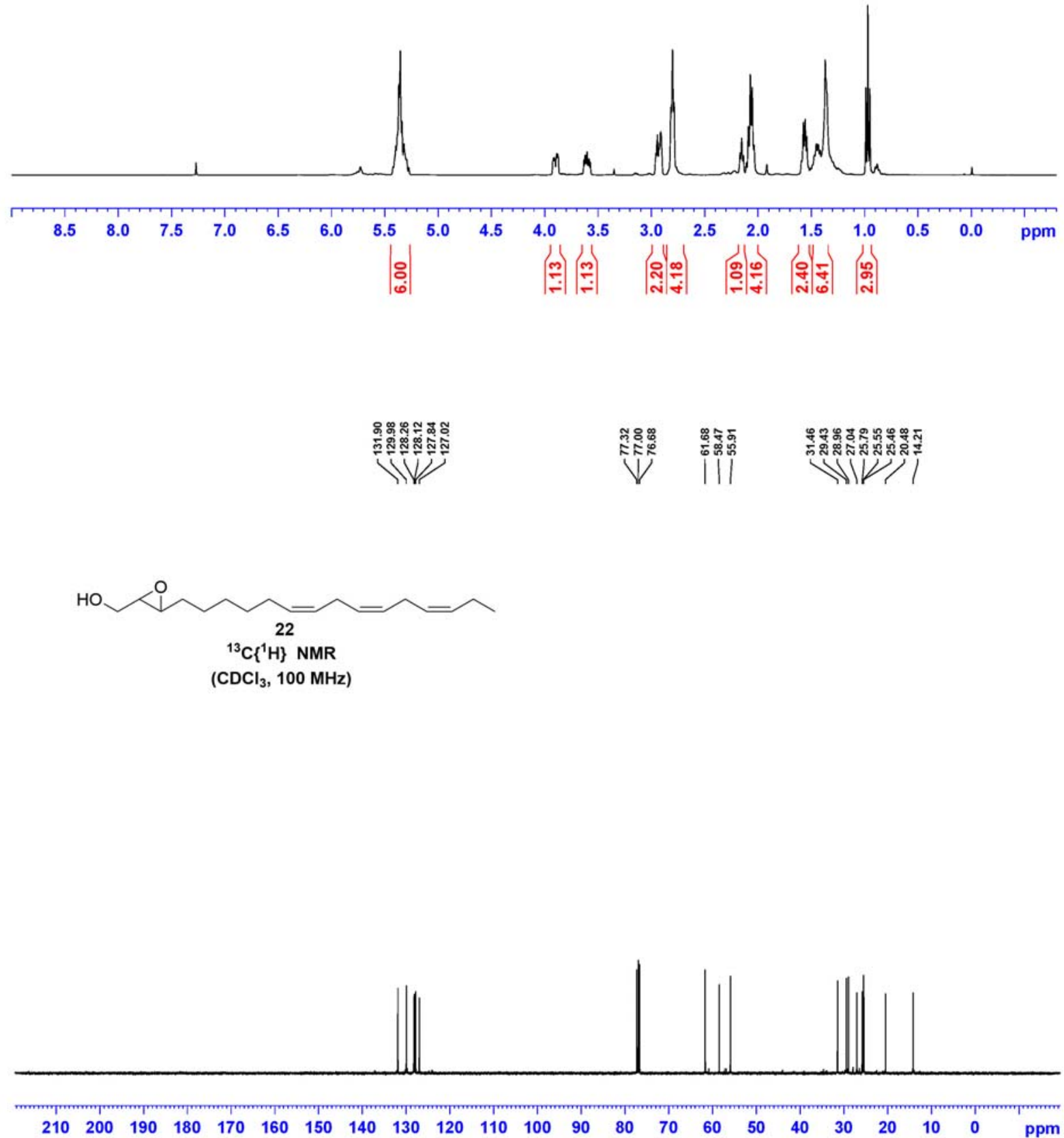

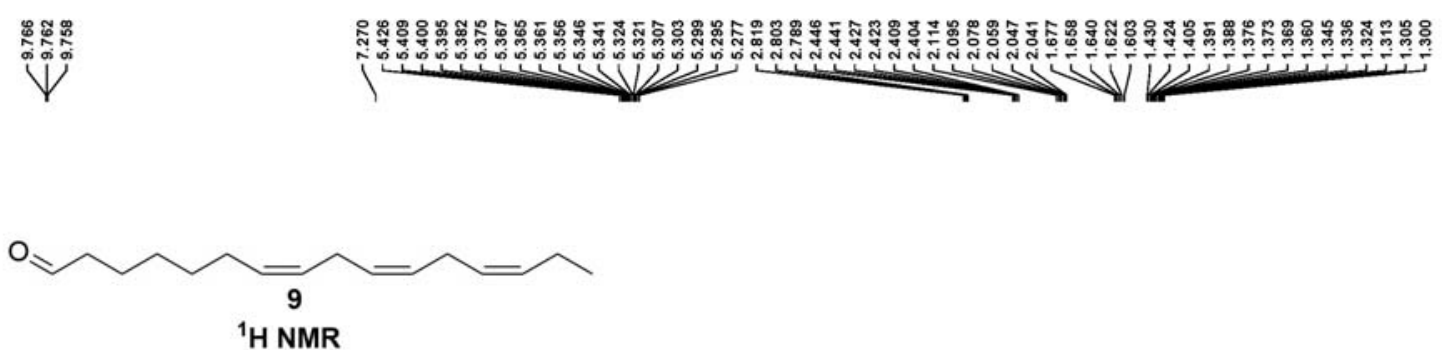

$\left(\mathrm{CDCl}_{3}, 400 \mathrm{MHz}\right)$
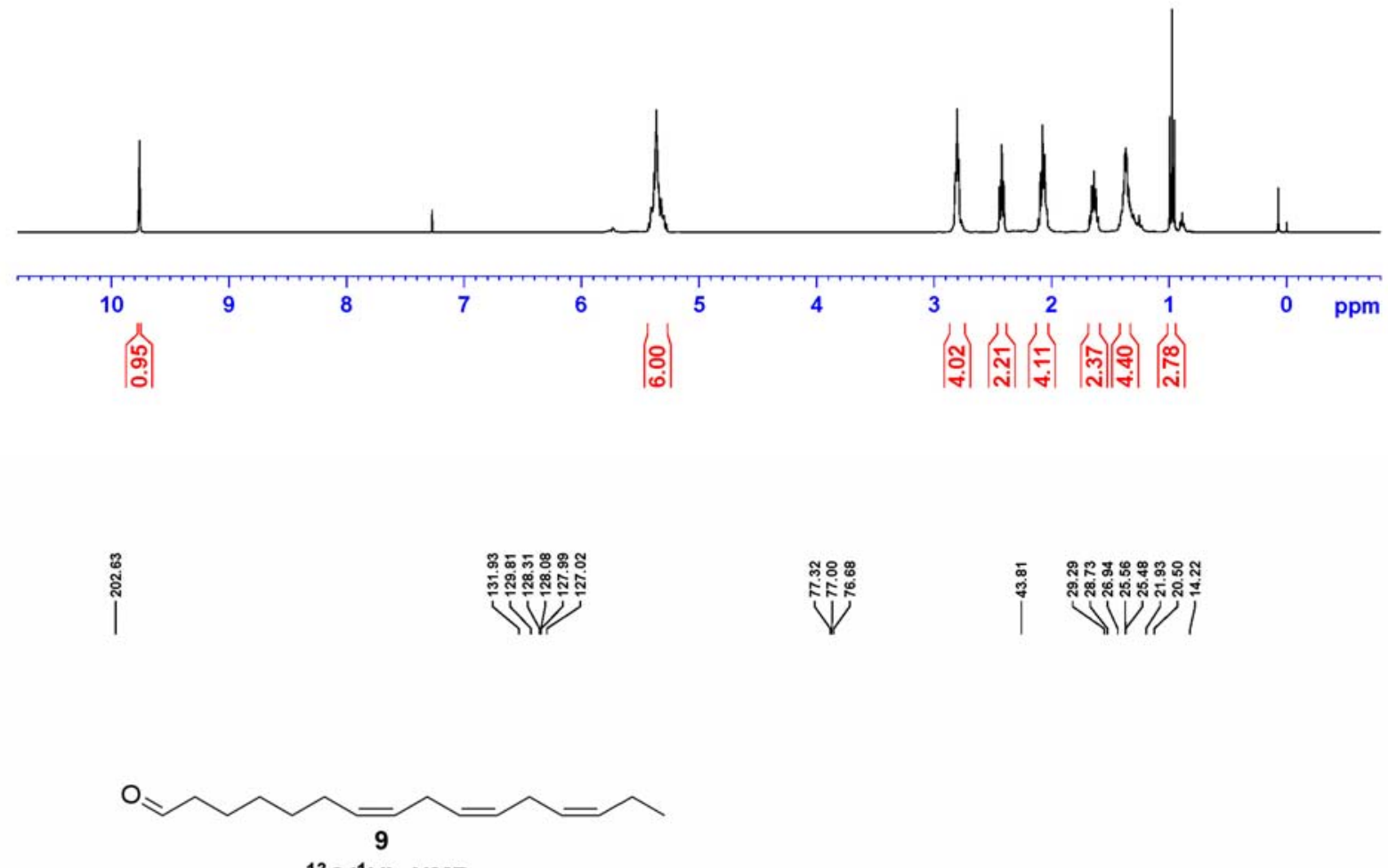

${ }^{13} \mathrm{C}\left\{{ }^{1} \mathrm{H}\right\}$ NMR

$\left(\mathrm{CDCl}_{3}, 100 \mathrm{MHz}\right)$

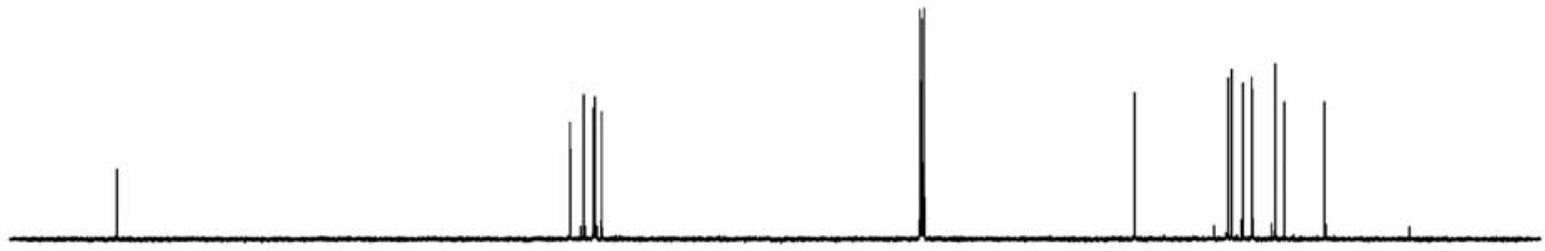

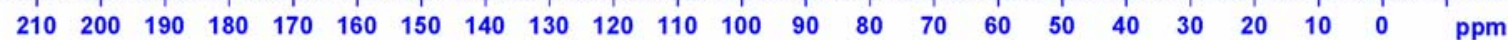




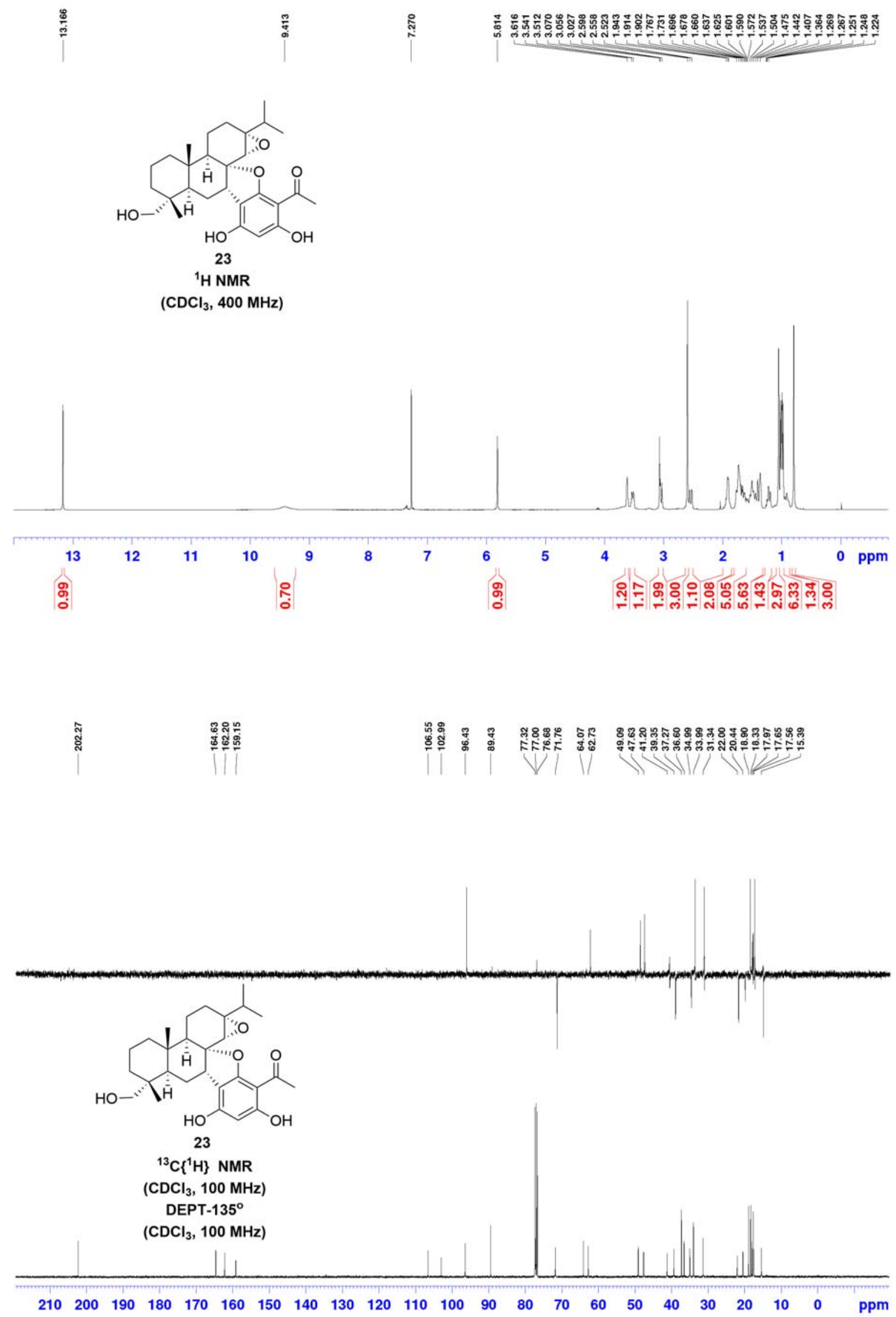


$\frac{\text { g }}{\stackrel{m}{m}}$

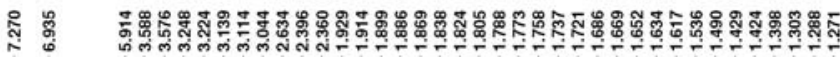

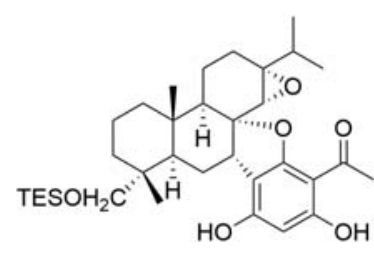

24

${ }^{1} \mathrm{H}$ NMR

$\left(\mathrm{CDCl}_{3}, 400 \mathrm{MHz}\right)$

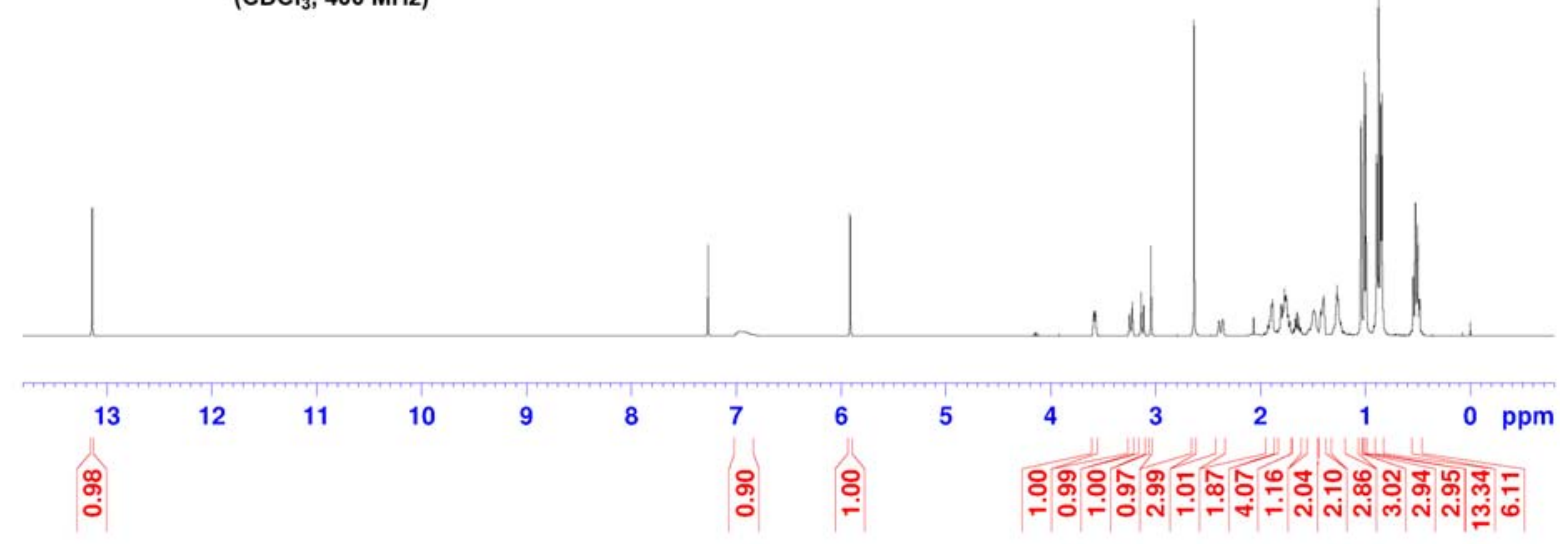

ণั่

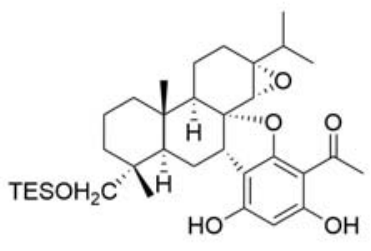

24

${ }^{13} \mathrm{C}\left\{{ }^{1} \mathrm{H}\right\}$ NMR

$\left(\mathrm{CDCl}_{3}, 100 \mathrm{MHz}\right)$

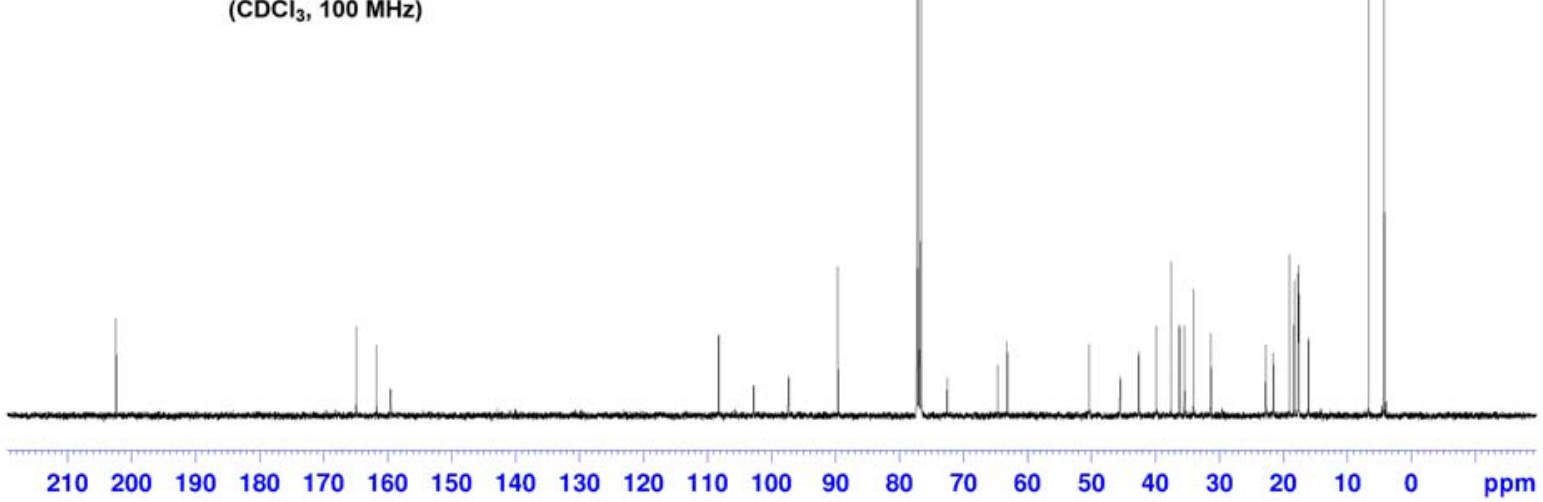




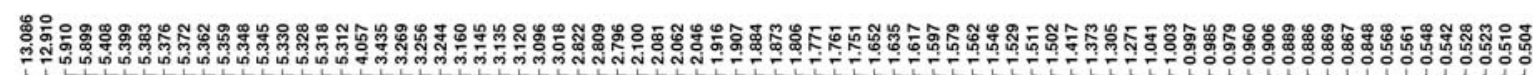
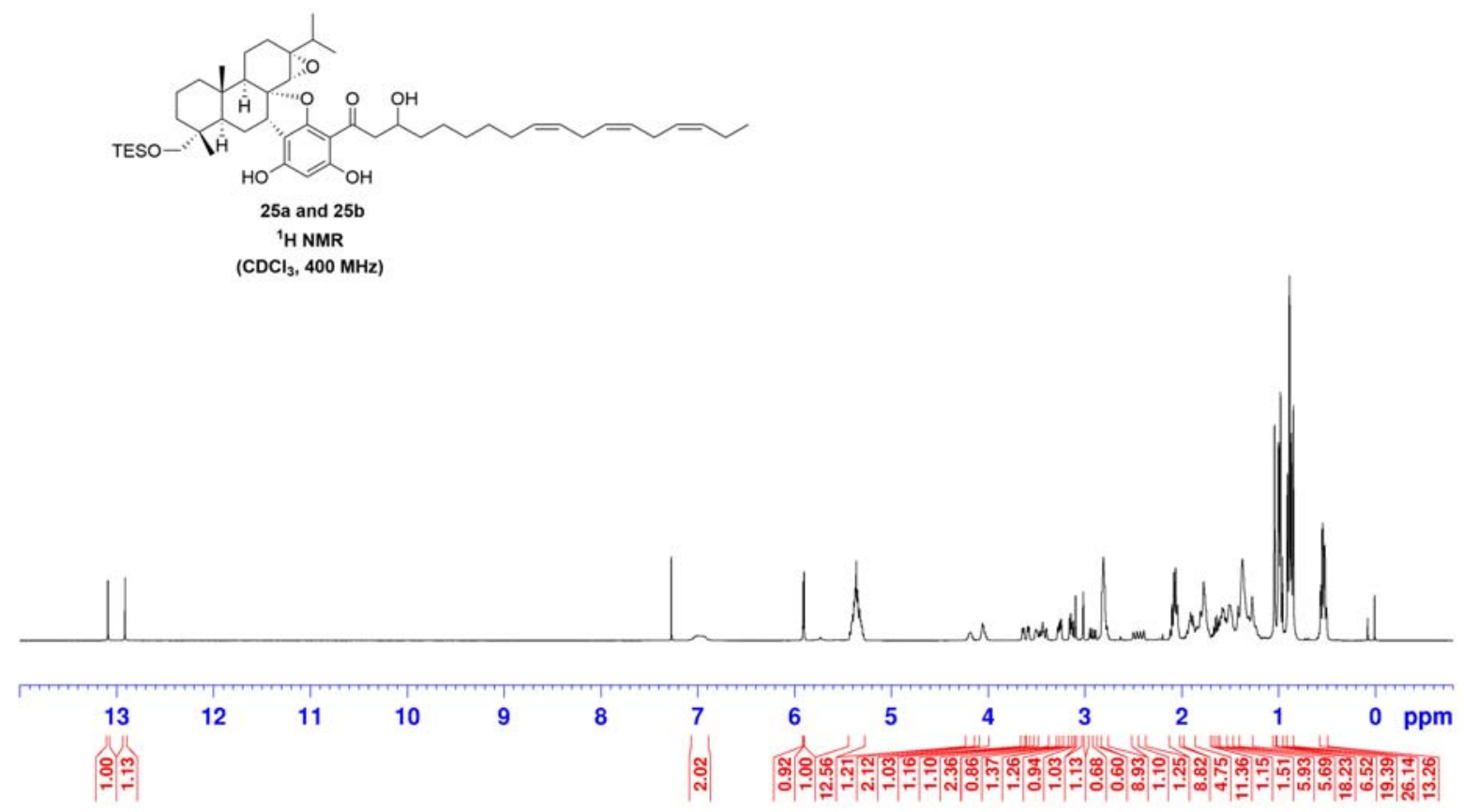

V
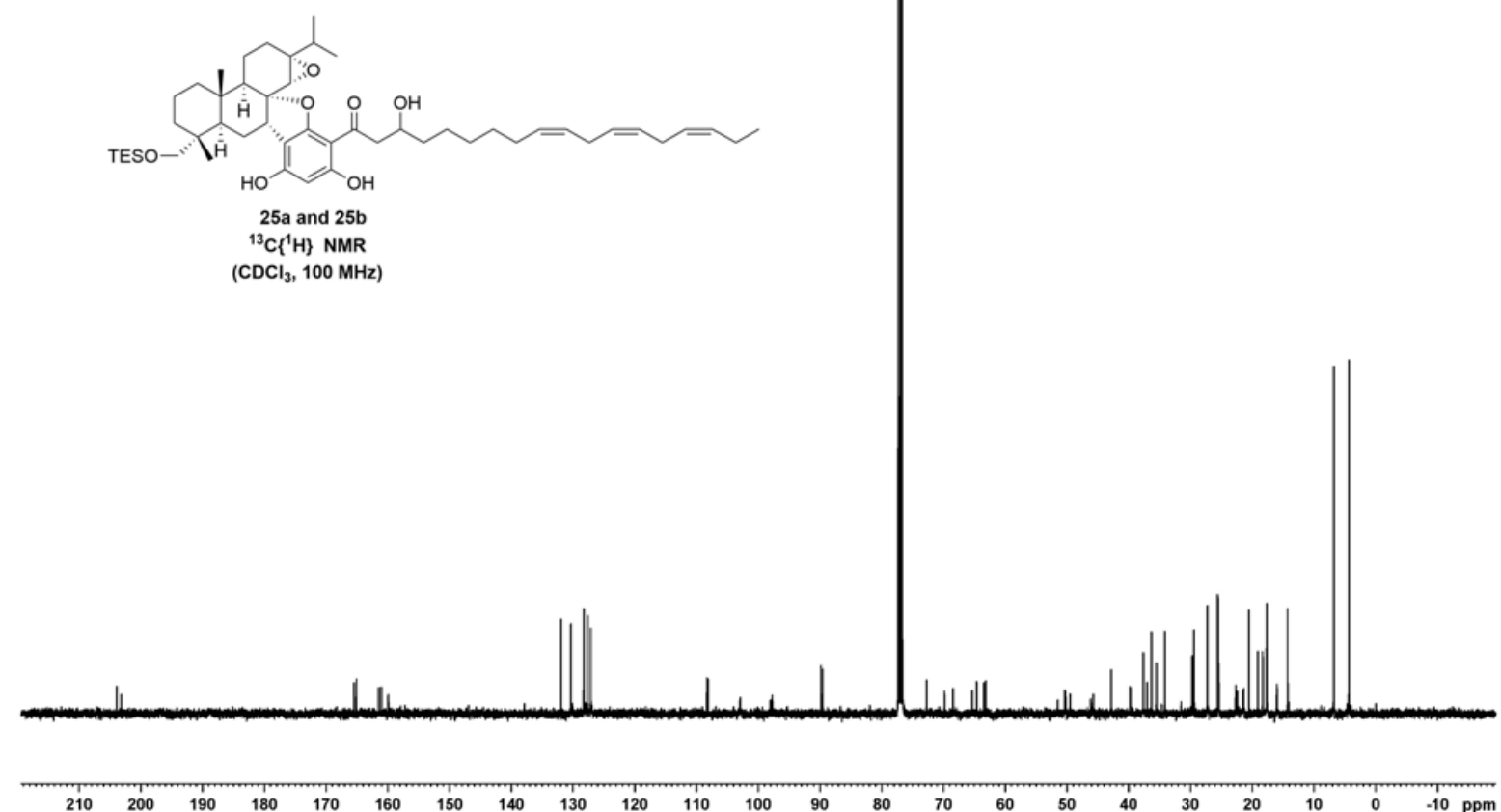


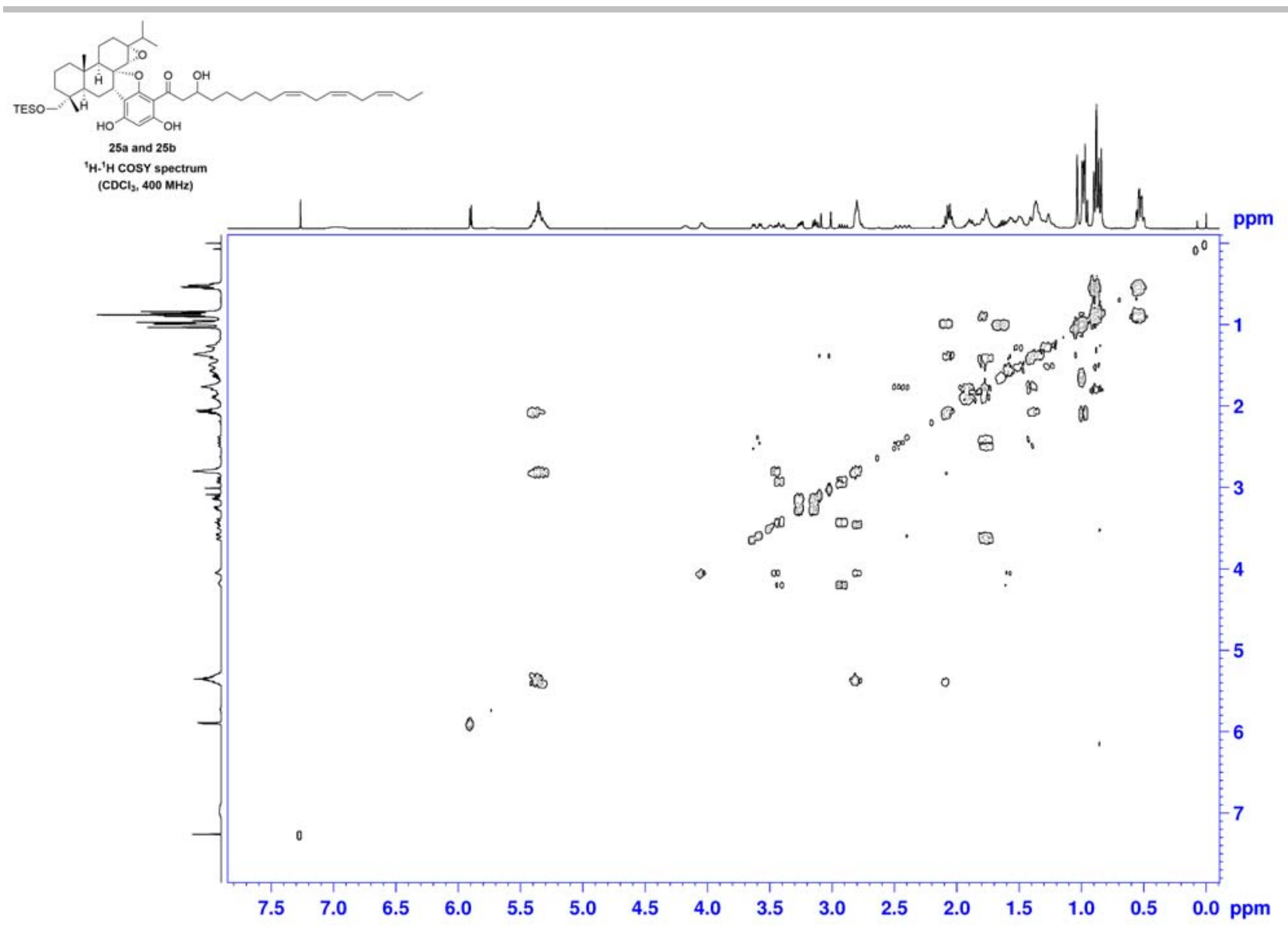

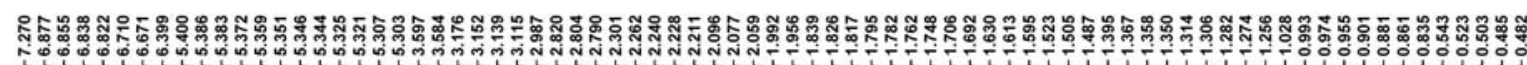

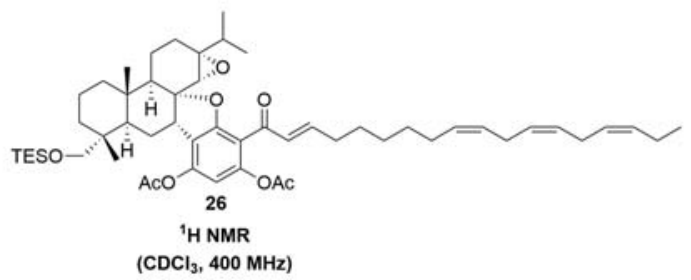

( $\mathrm{CDCl}_{3}, 400 \mathrm{MHz}$ )

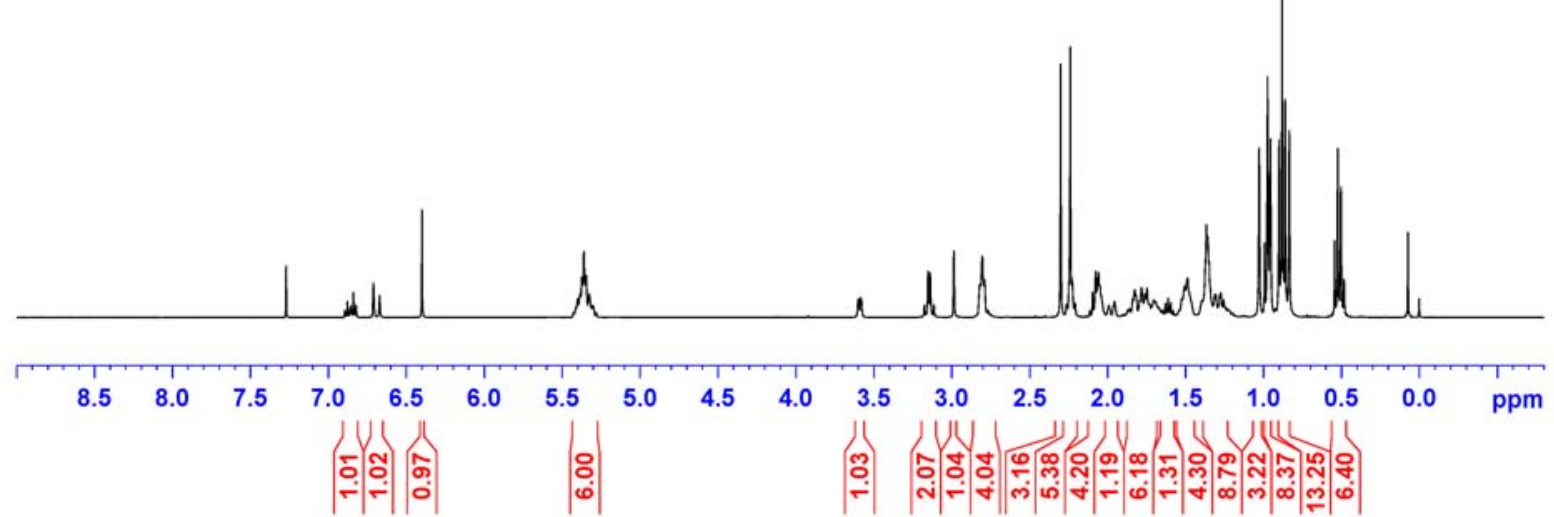



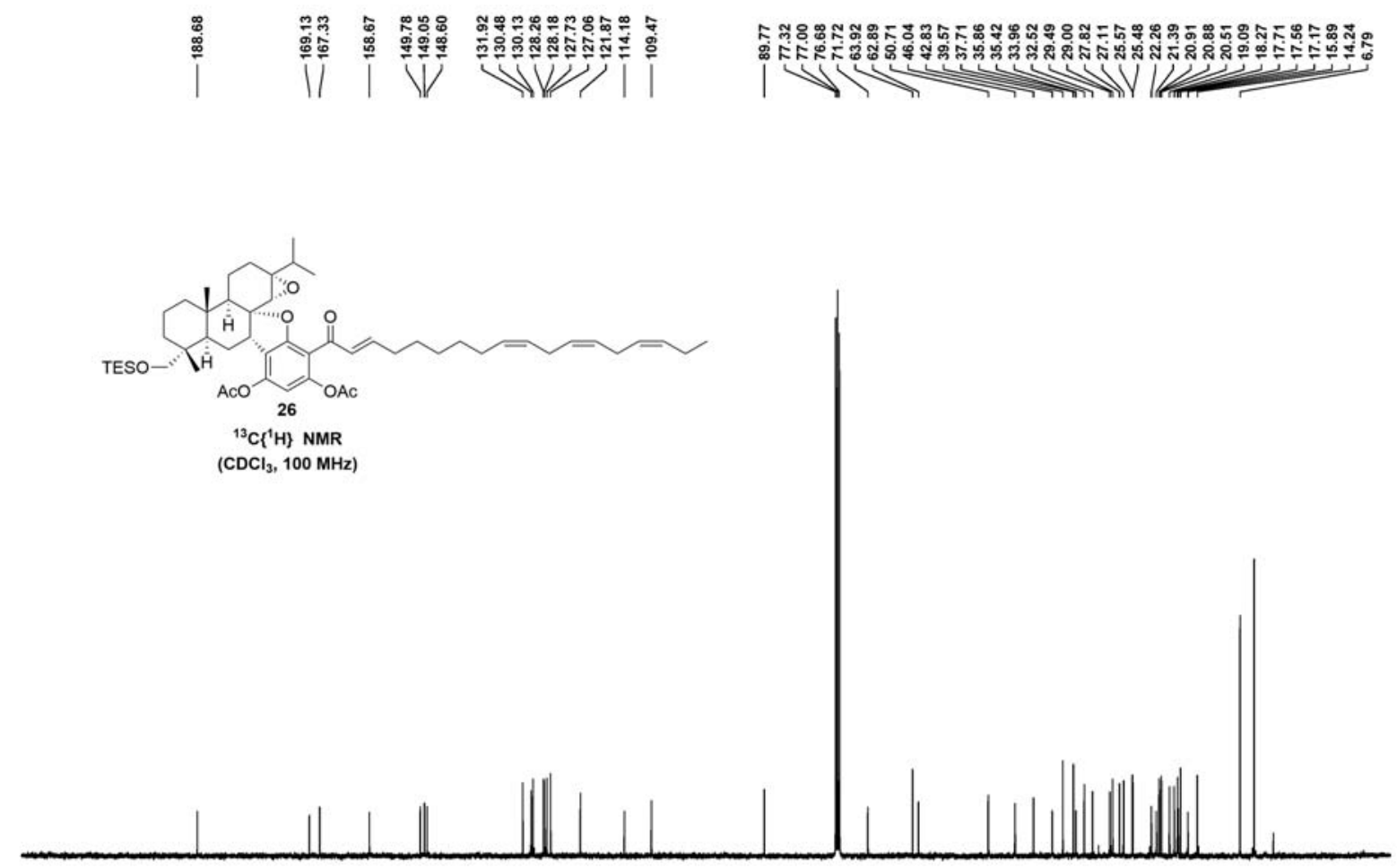

$\begin{array}{lllllllllllllllllllllllll}210 & 200 & 190 & 180 & 170 & 160 & 150 & 140 & 130 & 120 & 110 & 100 & 90 & 80 & 70 & 60 & 50 & 40 & 30 & 20 & 10 & 0 & \mathrm{ppm}\end{array}$

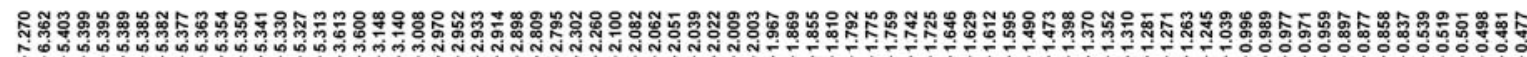

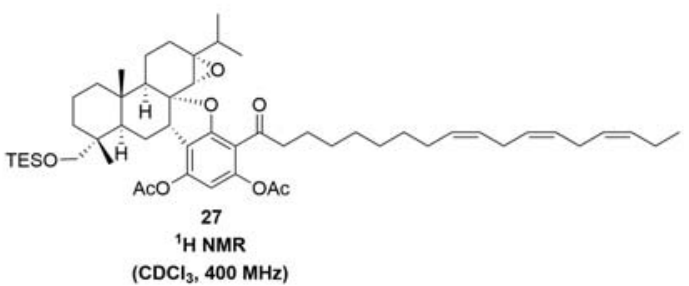

$\left(\mathrm{CDCl}_{3}, 400 \mathrm{MHz}\right)$

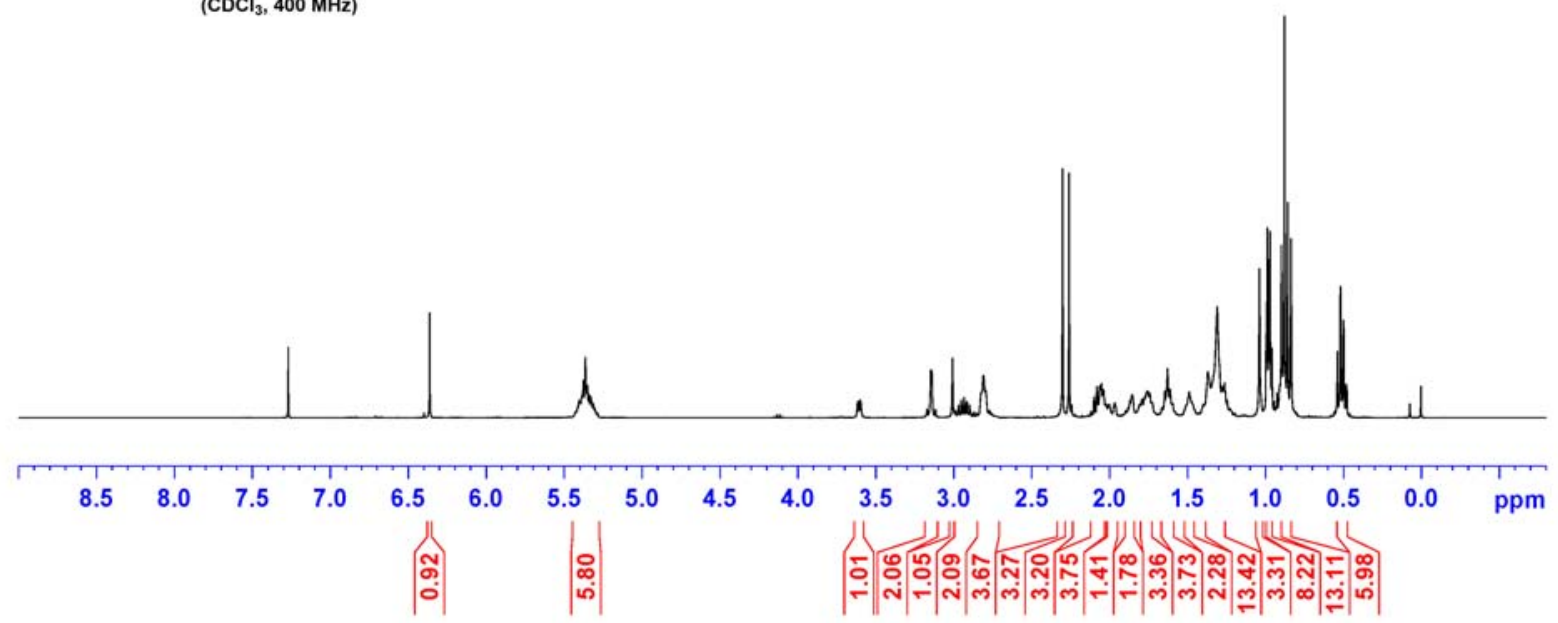




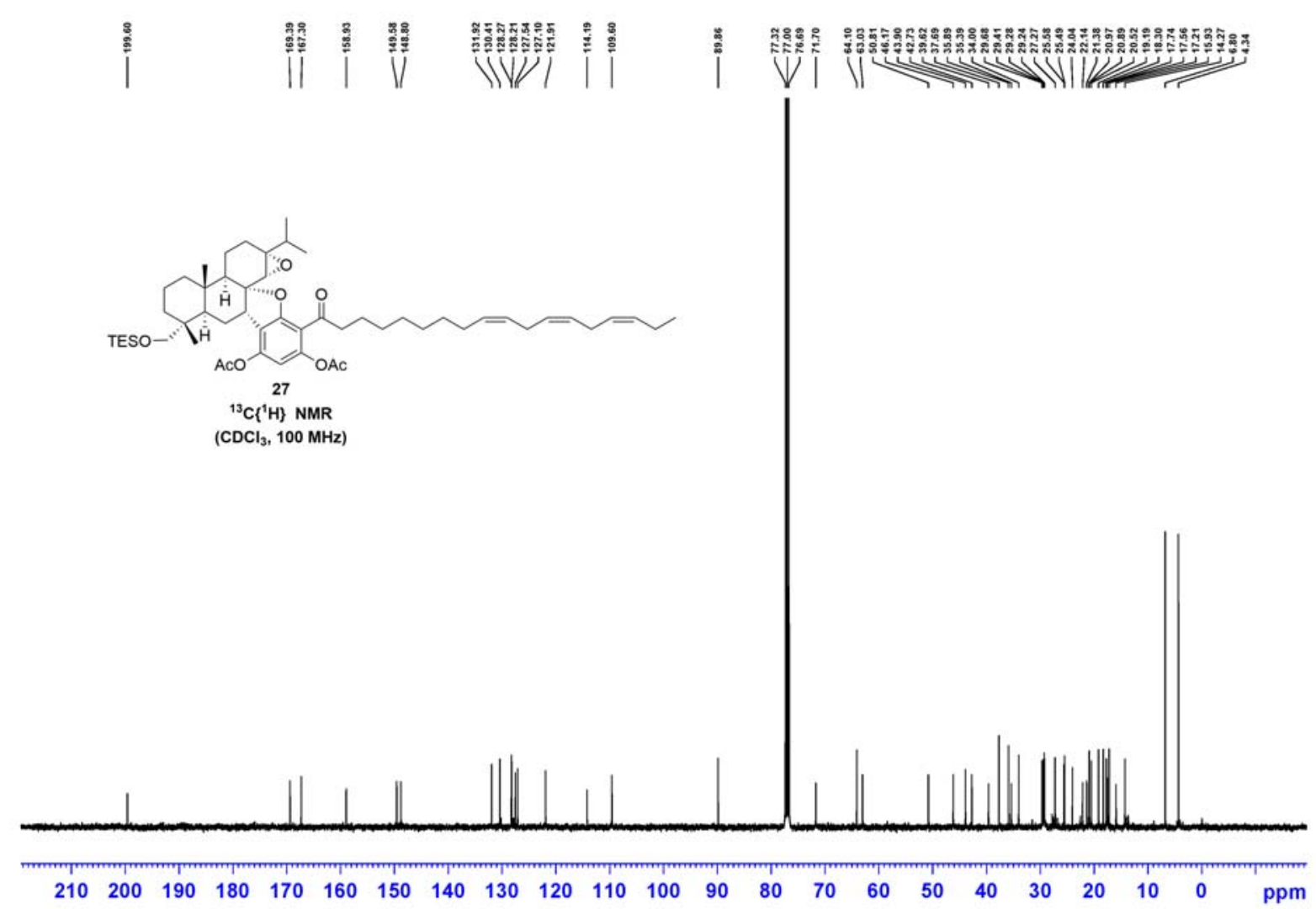

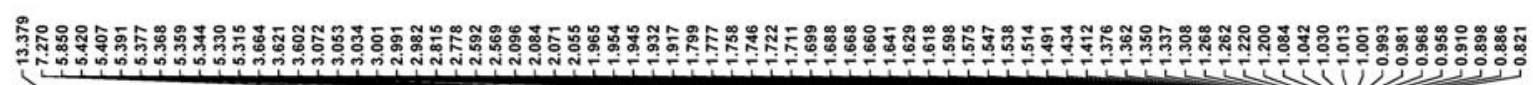
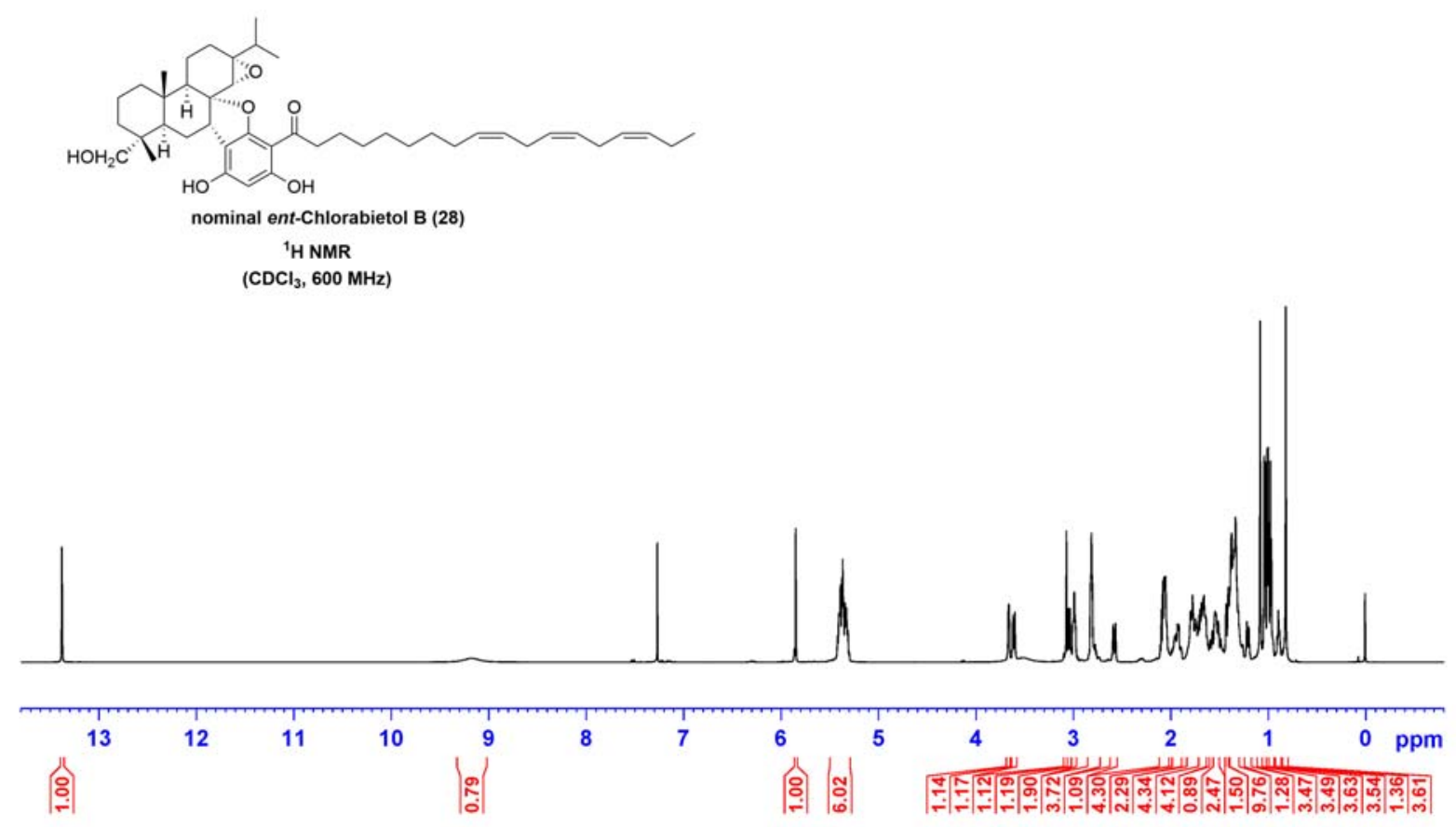

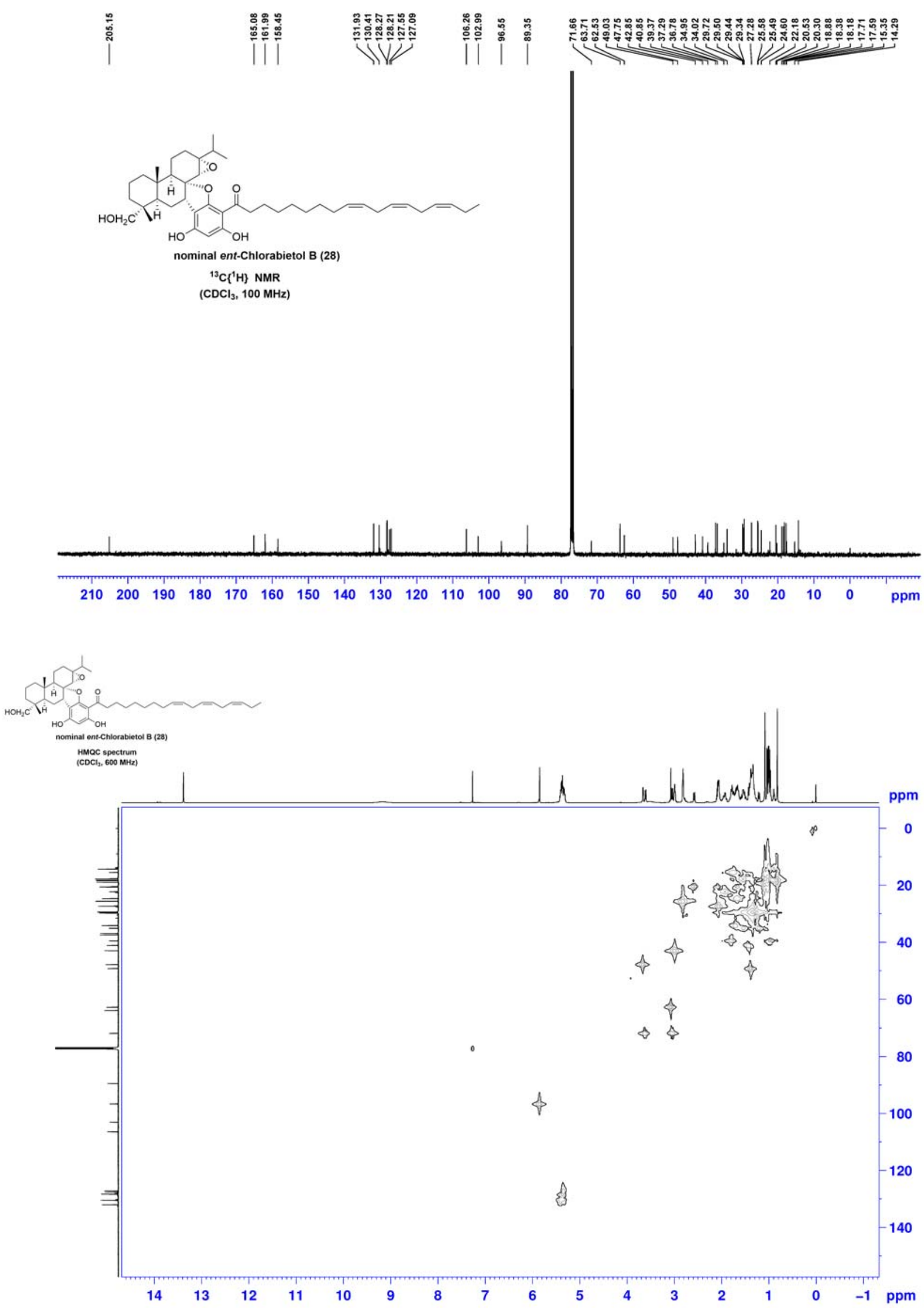


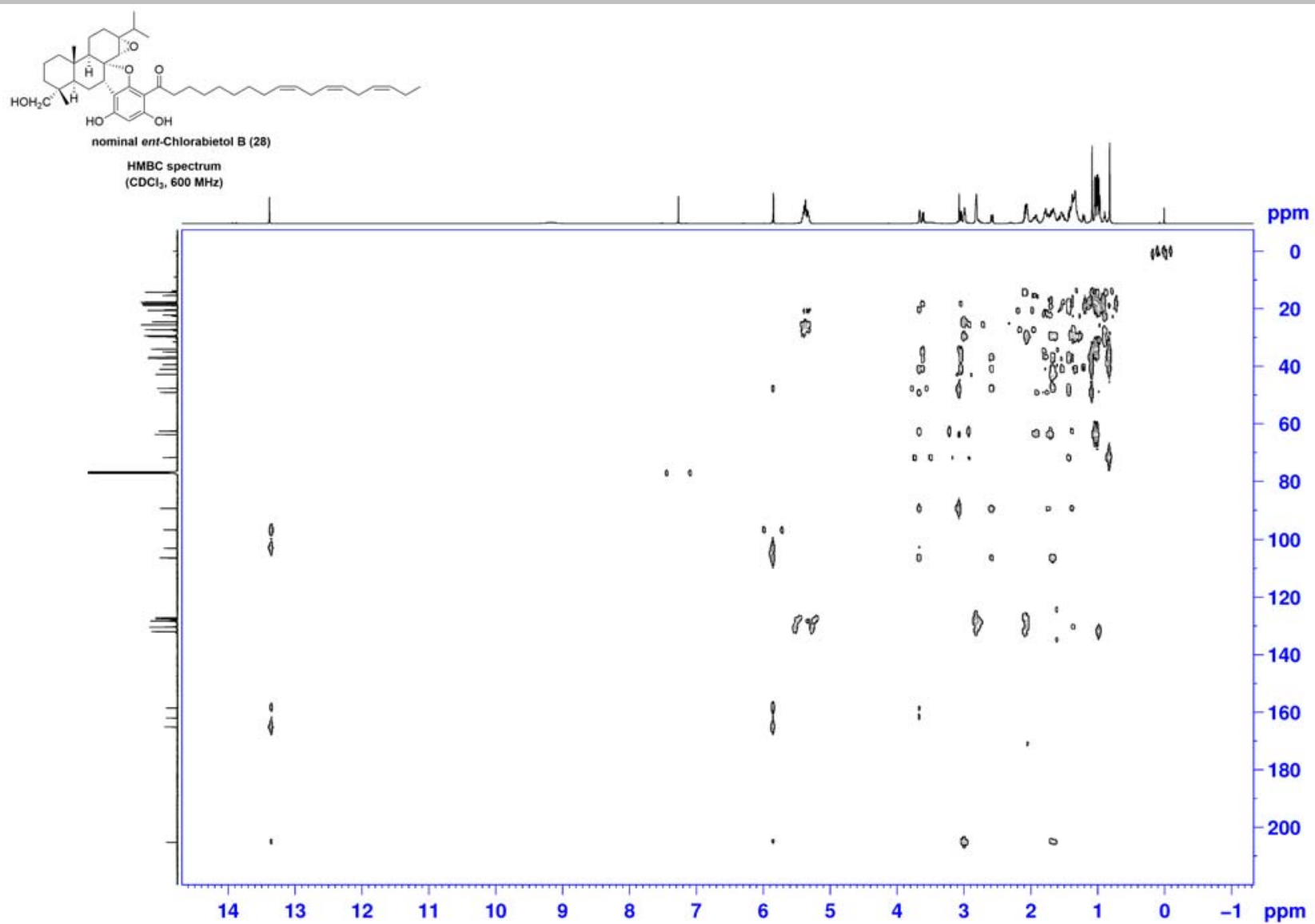




\section{NMR data comparison of synthetic sample and natural product}

NMR data comparison of synthetic sample and natural product

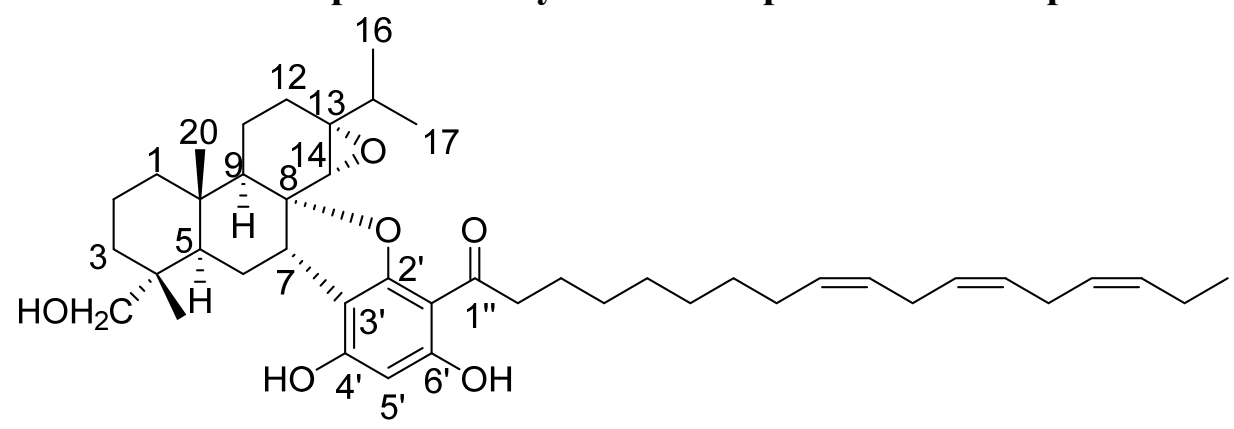

Table S2 ${ }^{1}$ H NMR Comparison

\begin{tabular}{|c|c|c|c|c|c|}
\hline & Natural & Synthetic & & Natural & Synthetic \\
\hline no. & 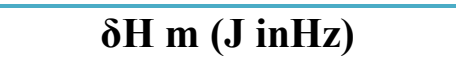 & $\delta_{H} \mathbf{m}(J$ inHz) & no. & $\delta_{H} m(J$ inHz) & \\
\hline $1 \alpha$ & $1.68, \mathrm{~m}$ & $1.81, \mathrm{~m}$ & 1 ' & & \\
\hline $1 \beta$ & 0.89, dd $(12.3,12.0)$ & $0.99, \mathrm{~m}$ & 2 & & \\
\hline $2 \beta$ & $1.59, \mathrm{~m}$ & $1.78, \mathrm{~m}$ & $3^{\prime}$ & & \\
\hline $2 \alpha$ & $1.49, \mathrm{~m}$ & $0.97, \mathrm{~m}$ & 4 ' & & \\
\hline $3 \alpha$ & 1.19, br d $(0.8)$ & $1.21, \mathrm{~d}(12)$ & 5 & $5.88, \mathrm{~s}$ & $5.85, \mathrm{~s}$ \\
\hline $\mathbf{3 \beta}$ & $1.50, \mathrm{dd}(12.0,10.8)$ & $1.50, \mathrm{~m}$ & 6' & & \\
\hline 4 & & & 1 ', & & \\
\hline $5 \beta$ & 1.42, br d (12.9) & $1.42, \mathrm{~d}(12.0)$ & 2 & $2.99, \mathrm{dt}(15.1,7.8)$ & $2.99, \mathrm{t}(6)$ \\
\hline $6 \beta$ & 2.47, br d $(14.7)$ & 2.58, br d $(12.0)$ & 3, & $1.66, \mathrm{~m}$ & $1.67, \mathrm{~m}$ \\
\hline $6 \alpha$ & 1.76, ddd $(14.7,12.9,5.2)$ & $1.66, \mathrm{~m}$ & $4 "$ & $1.33, \mathrm{~m}$ & $1.34, \mathrm{~m}$ \\
\hline $7 \alpha$ & 3.71 , br d (5.2) & 3.66, br d $(2.6)$ & 5, & $1.33, \mathrm{~m}$ & $1.34, \mathrm{~m}$ \\
\hline 8 & & & $6 "$ & $1.33, \mathrm{~m}$ & $1.34, \mathrm{~m}$ \\
\hline $9 \beta$ & 1.66, overlap & $1.38, \mathrm{~m}$ & 7, & $1.33, \mathrm{~m}$ & $1.34, \mathrm{~m}$ \\
\hline 10 & & & 8, & $2.06, \mathrm{~m}$ & $2.05, \mathrm{~m}$ \\
\hline $11 \alpha$ & $1.96, \mathrm{~m}$ & $1.58, \mathrm{~m}$ & 9', & $5.38, \mathrm{~m}$ & $5.35, \mathrm{~m}$ \\
\hline $11 \beta$ & 2.04, br d (12.0) & $1.61, \mathrm{~m}$ & $10 "$ & $5.31, \mathrm{~m}$ & $5.32, \mathrm{~m}$ \\
\hline 12 & $1.33,2 \mathrm{H}, \mathrm{m}$ & $1.91, \mathrm{~m}$ & 11, & $2.82, \mathrm{t}(6.0)$ & $2.82, \mathrm{~m}$ \\
\hline 13 & & & $12 \%$ & $5.36, \mathrm{~m}$ & $5.37, \mathrm{~m}$ \\
\hline $14 \alpha$ & $3.03, \mathrm{~s}$ & $3.07, \mathrm{~s}$ & 13 & $5.36, \mathrm{~m}$ & $5.37, \mathrm{~m}$ \\
\hline 15 & $1.58, \mathrm{~m}$ & $1.72, \mathrm{~m}$ & 14, & $2.82, \mathrm{t}(6.0)$ & $2.82, \mathrm{~m}$ \\
\hline 16 & $1.05, \mathrm{~d}(7.2)$ & $1.03, \mathrm{~d}(7.0)$ & 15 & $5.33, \mathrm{~m}$ & $5.39, \mathrm{~m}$ \\
\hline 17 & $0.98, \mathrm{~d}(7.2)$ & $1.01, \mathrm{~d}(6.8)$ & $16 "$ & $5.40, \mathrm{~m}$ & $5.39, \mathrm{~m}$ \\
\hline \multirow[t]{2}{*}{18} & $3.60, \mathrm{~d}(11.7)$ & $3.61, \mathrm{~d}(11.7)$ & $17^{\prime \prime}$ & $2.07, \mathrm{~m}$ & $2.08, \mathrm{~m}$ \\
\hline & $3.03, \mathrm{~d}(11.7)$ & $3.04, \mathrm{~d}(11.7)$ & & & \\
\hline 19 & $0.80, \mathrm{~s}$ & $0.81, \mathrm{~s}$ & 18 & $0.98, \mathrm{t}(7.5)$ & $0.98, \mathrm{t}(7.6)$ \\
\hline \multirow[t]{2}{*}{20} & $1.09, \mathrm{~s}$ & $1.08, \mathrm{~s}$ & 6'-OH & $13.4, \mathrm{~s}$ & $13.4, \mathrm{~s}$ \\
\hline & & & 4'-OH & & 9.17, brs \\
\hline
\end{tabular}


Table S3 ${ }^{13} \mathrm{C}$ NMR Comparison

\begin{tabular}{|c|c|c|c|c|c|}
\hline & Natural & Synthetic & & Natural & Synthetic \\
\hline $\begin{array}{l}\text { no. } \\
1 \alpha\end{array}$ & $\begin{array}{c}\delta_{\mathrm{c}} \\
39.9\end{array}$ & $\begin{array}{c}\delta_{\mathrm{c}} \\
39.4\end{array}$ & no. & $\begin{array}{c}\delta_{\mathrm{c}} \\
102.8\end{array}$ & $\begin{array}{c}\delta_{\mathrm{c}} \\
103.0\end{array}$ \\
\hline $1 \beta$ & & & 2 & 161.4 & 162.0 \\
\hline $2 \beta$ & 17.8 & 15.4 & 3 & 107.3 & 106.3 \\
\hline $2 \alpha$ & & & $4 '$ & 158.9 & 158.6 \\
\hline $3 \alpha$ & 35.2 & 35.0 & 5 & 96.9 & 96.5 \\
\hline $3 \beta$ & & & $6^{\prime}$ & 165.0 & 165.0 \\
\hline 4 & 37.6 & 37.3 & $1 '$ & 204.3 & 205.2 \\
\hline $5 \beta$ & 41.0 & 40.9 & 2, & 43.0 & 42.8 \\
\hline $6 \beta$ & 20.7 & 20.3 & 3 ', & 24.1 & 24.6 \\
\hline $6 \alpha$ & & & 4, & 29.3 & 29.3 \\
\hline $7 \alpha$ & 46.6 & 47.7 & 5, & 29.4 & 29.4 \\
\hline 8 & 92.7 & 89.4 & $6 "$ & 29.7 & 29.5 \\
\hline $9 \beta$ & 51.6 & 49.1 & $7 '$ & 29.7 & 29.7 \\
\hline 10 & 37.3 & 36.8 & $8^{\prime \prime}$ & 27.3 & 27.3 \\
\hline $11 \alpha$ & 21.6 & 18.9 & 9', & 130.3 & 130.4 \\
\hline $11 \beta$ & & & 10 ', & 127.1 & 127.1 \\
\hline 12 & 29.7 & 22.0 & 11 ', & 25.6 & 25.6 \\
\hline 13 & 64.0 & 63.7 & $12 "$ & 128.2 & 128.2 \\
\hline $14 \alpha$ & 62.3 & 62.6 & 13 " & 128.3 & 128.3 \\
\hline 15 & 34.5 & 34.0 & 14 " & 25.5 & 25.5 \\
\hline 16 & 17.5 & 17.6 & 15, & 127.7 & 127.6 \\
\hline 17 & 18.0 & 17.7 & $16 "$ & 132.0 & 131.9 \\
\hline 18 & 71.8 & 71.7 & 17, & 20.5 & 20.5 \\
\hline 19 & 18.0 & 18.1 & 18 ", & 14.3 & 14.3 \\
\hline 20 & 16.8 & 18.4 & & & \\
\hline
\end{tabular}

